\title{
Theoretical issues with staggered fermion simulations
}

\author{
Stephan Dürr*t \\ Bern University, ITP \\ Sidlerstrasse 5, 3012 Bern, Switzerland \\ E-mail: durrATitp.unibe.ch
}

The legality of the "rooting trick" in dynamical staggered fermion simulations is discussed, i.e. whether the theory with the Boltzmann weight $\operatorname{det}^{1 / 4}\left(D_{\text {st }}\right)$ yields the right continuum limit. Since the problem is unsolved, pieces of evidence in favor and against are collected and examined.

XXIIIrd International Symposium on Lattice Field Theory

25-30 July 2005

Trinity College, Dublin, Ireland

\footnotetext{
* Speaker.

${ }^{\dagger}$ Based on work done in collaboration with Christian Hoelbling (Wuppertal) and Urs Wenger (DESY Zeuthen).
} 


\section{Introduction}

Is "staggered QCD” really QCD, or is it just a model of QCD ? This is the question addressed in this note, and it is worth emphasizing two points right now: the issue is whether the staggered approach yields the right continuum limit and the question is asked at the non-perturbative level.

The staggered action is not doubler-free; the Kogut-Susskind construction manages to reduce the fermion multiplicity from 16 to 4 [1]. To simulate QCD with $N_{f}=2+1$ dynamical quarks staggered studies employ the "rooting trick", i.e. the Boltzmann weight used in the simulation is

$$
e^{-S_{\text {eff }}}=\operatorname{det}^{1 / 2}\left(D_{\text {st }, m_{u d}}\right) \operatorname{det}^{1 / 4}\left(D_{\text {st }, m_{s}}\right) e^{-S_{G}}
$$

where $m_{u d}=\left(m_{u}+m_{d}\right) / 2$ is an average up and down quark mass, $m_{s}$ is kept at the physical strange quark mass and $S_{G}$ denotes the gauge action. Throughout, I use the abbreviation $D_{\text {st }, m}=D_{\text {st }}+m$ for the massive staggered Dirac operator. The "rooting trick" is the way how the unphysical "tastes" (see below) are excised from the sea sector of the theory, and the issue of debate is whether the effective action may be re-expressed in terms of an artificial undoubled formulation which is local. Ideally, this construction would hold exactly on typical backgrounds, but in view of the first point emphasized above it might be $\mathrm{OK}$ if one can prove that a slight mismatch in the determinants is just a cut-off effect. I will explain below why it is so useful to have a local underlying theory. On the level of the effective action the question is whether multi-plaquette correlators have unphysical cuts in the limit where the lattice spacing $a$ is sent to zero.

The staggered action not being doubler-free has an impact on the valence sector, too. Here, sophisticated reduction techniques have been developed (already in the quenched era) to project out the desired spinor $\otimes$ taste combination from a given staggered operator. The problem is that the way how the unphysical tastes are excised from the valence sector is (in general) slightly different from the rooting procedure employed in the sea sector. Evidently, this means that at finite lattice spacing the theory is not unitary. Again, this does not necessarily lead to a disaster, if one can show that the non-unitarity is a genuine cut-off effect.

Personally, I tend to see the locality as the crucial issue and the unitarity as a point of lesser severity. This is so, because there are reasons to believe that a solution to the first problem would automatically provide a bypass to the second one, as sketched below.

The controversy, as I see it, is thus a debate whether the rooting procedure in the sea sector would introduce spurious degrees of freedom which corrupt, at the non-perturbative level, the longrange properties of the effective action in such a way that something survives the continuum limit. As of now, the answer is not known. All I can provide is an attempt to explain basic facts needed to understand the origin of the problem and then collect, in a second step, pieces of evidence in favor and against the legality of the staggered approach. It turns out that the evidence "in favor" outnumbers the one "against" by far, but in the end the sheer number has no say.

A key issue in practical applications of staggered fermions is to offset the taste symmetry breaking as much as possible. In the course of the simulation this is done by using improved/filtered varieties of the staggered action which may reduce the taste splitting quite drastically (see below). In the analysis of pseudo-scalar meson properties Staggered Chiral Perturbation Theory (SXPT) is used, an effective field theory that allows one to parameterize and thus "take out" the leading taste 
breaking effects. Many of the pieces of evidence to be presented below will use one or the other strategy to tame the taste symmetry breaking. Still, from a fundamental viewpoint it is clear that either one of these techniques is immaterial - if the staggered approach is correct, it will yield the right continuum limit for arbitrary observables without any of these.

Obviously, there are two ways how the controversy could play out. One possible outcome is realized, if someone manages to give an unambiguous (mathematical) proof that QCD with $N_{f}=2+1$ staggered quarks is in the right universality class. The other option is, of course, that someone manages to find a single observable where he/she can demonstrate that the staggered answer, after a continuum extrapolation, is wrong.

Before getting into details, let me comment on one trap to be avoided. It is true that some of the more recent studies with $N_{f}=2+1$ dynamical staggered quarks agree (in particular in observables relating to $\Upsilon$ spectroscopy) with experiment to a precision that is currently not matched by another fermion discretization (see e.g. [2]). Still, from a conceptual viewpoint this is no help in deciding whether the staggered approach is fundamentally correct or "epicycle physics". In particular the agreement of a mixed QCD/electroweak observable with experiment is not really an argument, since what we want to find out is whether there is a contribution from beyond-the-SM physics in such observables. What comparing to experiment is supposed to tell us is whether the Lagrangian relevant to a problem is the one we stick into our code, and for this the method itself has to be from first principles. Hence, whether QCD with $N_{f}=2+1$ staggered fields is fundamentally correct is an issue that needs to be solved theoretically - by pure thought or with the help of CPU power.

The plan is to first review some elements of the staggered action and taste representation. The question about the legality of the "rooting trick" is then exposed as a locality problem that would be solved if one could find a "candidate" operator that satisfies certain criteria. In the free theory the search has ended successfully, and I discuss the four constructions known to date. For the interacting theory I assess pieces of evidence that come from studying spectral and eigenmode properties of the staggered action in $4 \mathrm{D}$. For many observables in 2D it is easy to reach good statistical precision and this leads to a somewhat more mixed picture in the Schwinger model. Still, just comparing the staggered and the overlap contribution to the effective action [i.e. correlating $-\frac{1}{2} \log \operatorname{det}\left(D_{\mathrm{st}, m}\right)$ and $-\log \operatorname{det} D_{\mathrm{ov}, m}$ in a scatter plot for $m>0$ ] lets one ask whether $D_{\mathrm{ov}, m}$ might actually represent (one version of) the desired "candidate" action. Finally, a few words on what can be learned from analyzing data with Staggered Chiral Perturbation Theory are in place. The problem being unsolved, I refrain from drawing any conclusion and end with a summary.

\section{Review: Staggered action and taste representation}

The staggered action derives from the naive fermion discretization

$$
S_{\mathrm{na}}=\frac{a^{3}}{2} \sum_{x, \mu} \bar{\psi}(x) \gamma_{\mu}\left[U_{\mu}(x) \psi(x+\hat{\mu})-U_{\mu}^{\dagger}(x-\hat{\mu}) \psi(x-\hat{\mu})\right]
$$

which is known to lead to 4 (in 2D) or 16 (in 4D) fermions in the continuum ( $2^{d}$ species in $d$ dimensions). The observation by Kogut and Susskind was that the $x$-dependent field transformation 
$\psi(x)=\gamma(x) \chi(x), \bar{\psi}(x)=\bar{\chi}(x) \gamma(x)^{\dagger}, \gamma(x)=\gamma_{1}^{n_{1}} \gamma_{2}^{n_{2}} \gamma_{3}^{n_{3}} \gamma_{4}^{n_{4}}$ with $x=n a$ and the staggered phase factor $\eta_{\mu}(x)=(-1)^{\sum_{\mu<v} n_{v}}$ brings it to the form

$$
S_{\mathrm{na} / \mathrm{st}}=\frac{a^{3}}{2} \sum_{x, \mu} \bar{\chi}(x) \eta_{\mu}(x)\left[U_{\mu}(x) \chi(x+\hat{\mu})-U_{\mu}^{\dagger}(x-\hat{\mu}) \chi(x-\hat{\mu})\right]
$$

where the 2 (in 2D) or 4 (in 4D) components of $\chi$ (in general: $2^{d / 2}$ ) manifestly decouple. Therefore, by just "downgrading" $\chi$ to be a 1-component field, the action (2.2) is changed to the staggered one which yields only $N_{t}=2^{d / 2}$ fermions in the continuum. The Kogut Susskind procedure thus manages to "thin out" degrees of freedom, albeit at the price of distributing/intertwining "spinor" and "taste" (the latter being the modern word to address the full fermion content of a staggered field). An important point is that the $S U\left(N_{t}\right)_{A}$ taste symmetry in the formal continuum limit reduces to the abelian invariance $\chi(x) \rightarrow e^{\mathrm{i} \theta_{A} \eta_{5}(x)} \chi(x), \bar{\chi}(x) \rightarrow e^{-\mathrm{i} \theta_{A} \eta_{5}(x)} \bar{\chi}(x)$ of the massless action at finite lattice spacing, and this is good enough to prevent additive mass renormalization. However, further exact "thinning" by purely algebraic means is impossible, since the Kogut Susskind procedure has exhausted the eigenvalue degeneracy of the naive action (the latter has only $2^{d / 2}$-fold degenerate eigenvalues on typical backgrounds, not $2^{d}$-fold, as one might have naively guessed).

Modern staggered simulations use an individual staggered field for each continuum flavor to be studied, i.e. QCD with $N_{f}=2+1$ quarks requires the $\chi$-fields $u, d, s$. The taste structure of physical operators is non-trivial $[3,4]$. The underlying taste identification may be attempted in momentum space [5] or in coordinate space [6, 7, 8]. The latter version builds on the hypercubic decomposition $\chi(x, x+a \hat{1}, \ldots, x+a \hat{1}+a \hat{2}+a \hat{3}+a \hat{4}) \rightarrow q(X)$ which collects all spinor/taste information in a $2^{4}$ sublattice (of the original one) into a "masternode", i.e. a point in the blocked lattice with spacing $b=2 a$. Denoting these coarse points with uppercase symbols, the free action reads

$$
S_{\mathrm{st}}=b^{4} \sum_{X, \mu} \bar{q}(X)\left[\nabla_{\mu}\left(\gamma_{\mu} \otimes I\right)-\frac{b}{2} \triangle_{\mu}\left(\gamma_{5} \otimes \tau_{\mu} \tau_{5}\right)\right] q(X)
$$

where $q(X)$ is a 16-component "quark field" (in 4D) and the first and second derivative operate on the blocked lattice, i.e. $\left(\nabla_{\mu} q\right)(X)=\frac{q(X+b \hat{\mu})-q(X-b \hat{\mu})}{2 b}$ and $\left(\triangle_{\mu} q\right)(X)=\frac{q(X+b \hat{\mu})-2 q(X)+q(X-b \hat{\mu})}{b^{2}}$. The tensor structure in (2.3) refers to spinor $\otimes$ taste, where $\tau_{\mu}=\gamma_{\mu}^{*}, \tau_{5}=\gamma_{5}$, i.e. another set of gamma matrices is used to map out the taste algebra. The main observation is that in this basis the troublesome taste changing interactions stem from a dimension 5 Wilson-type term which is "skewed" in spinor $\otimes$ taste space (and hence not positive semidefinite).

The case of interest is the interacting theory, and here the question is whether all taste changing effects would go away, in the continuum limit, without leaving any trace. Of course, from a formal viewpoint this seems almost obvious, since it is a dimension 5 piece ${ }^{1}$ in (2.3) that mediates the taste changes, and irrelevant operators have no anomalous dimensions. As pointed out in [10] the whole issue is a peculiar exchange-of-limits question. If the formal continuum limit is justified (and the view that naturally derives from the taste basis thus adequate), then everything is fine, indeed. The problem is just that one would need to prove first that the staggered action yields the correct continuum limit to be sure that adopting this viewpoint is not misleading.

\footnotetext{
${ }^{1}$ As worked out by Luo the $O(a)$ term in (2.3) can be shifted to $O\left(a^{2}\right)$ by a change of basis [9].
} 

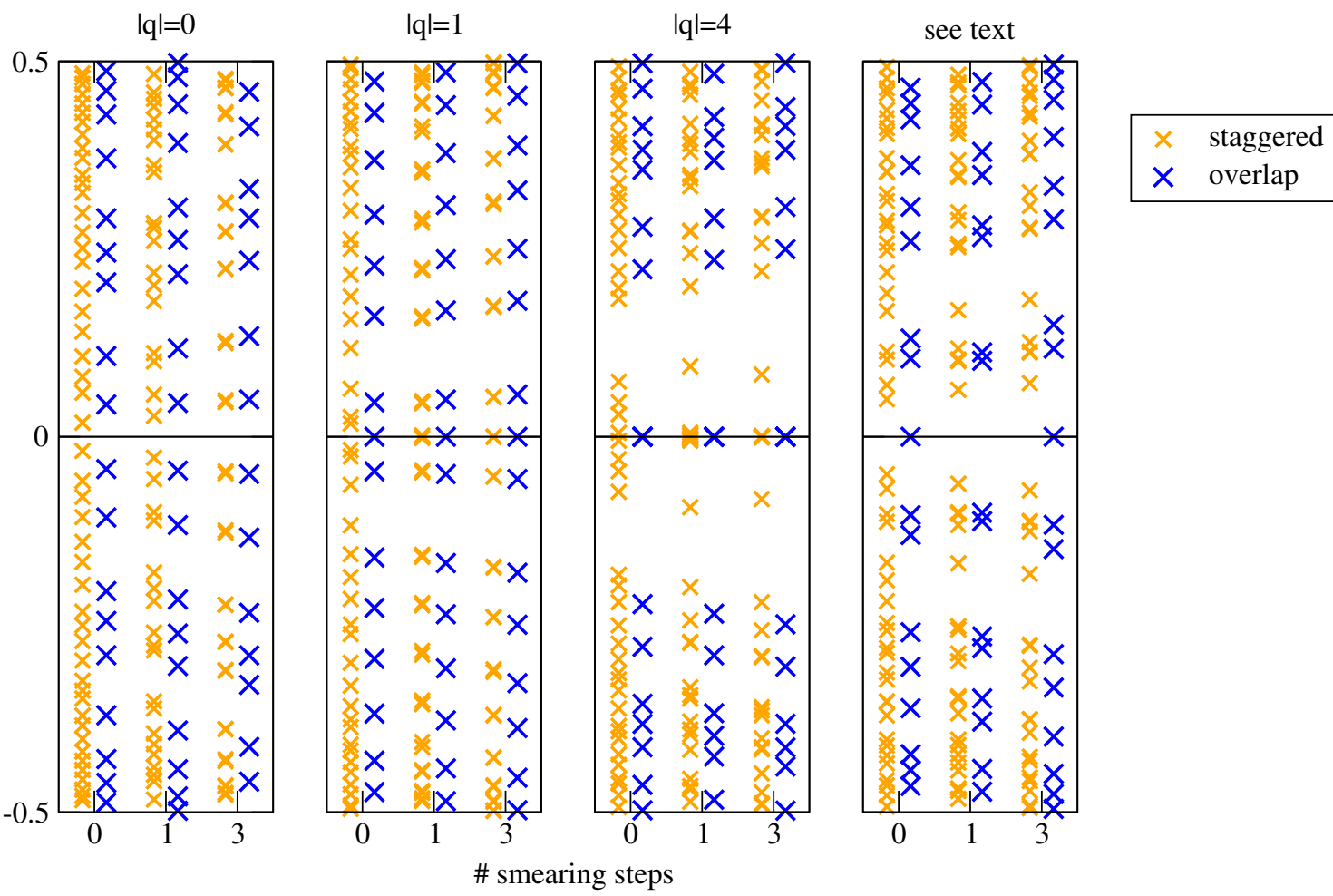

Figure 1: Staggered and overlap eigenvalues on 4 selected configurations in 2D. On benevolent configurations the improved taste symmetry achieved through filtering results in a 2-fold (4-fold in 4D) neardegeneracy and the right number of near-zero modes. To aid comparison the low-energy overlap spectrum has been mapped onto the imaginary axis with a stereographic projection. Figure from [39].

One way to think about taste changing interactions is to notice that in the interacting theory individual taste (or spinor) components of a $q$-field still sit out there in the little $2^{4}$ hypercube attached to a blocked "masternode". They see slightly different local gauge fields (therefore spinor $\otimes$ taste projection operators must be gauged, e.g. the staggered $\gamma_{5}$ is a 4-link operator). Equivalently, in the momentum space view each spinor/taste component has a reduced Brillouin zone with extension $2 \pi / b=\pi / a$ in each direction and this means that hard gluons with one component $\sim \pi / a$ will flip one taste to another one. In any case it is clear that the identification of physical and staggered flavor may only work, if taste interactions are minimized (ideally: eliminated).

Everything below will hinge on this taste symmetry (which at finite lattice spacing is only approximate), and Fig. 1 is an attempt to convey a down-to-earth view. If the taste symmetry were exact the staggered eigenvalue spectrum would show a 2-fold (in 2D) or 4-fold (in 4D) degeneracy. On typical configurations smearing (i.e. using a filtered fermion operator, see below) is a convenient tool (read: cheaper than decreasing $a$ ) to achieve a rather good $N_{t}$-fold near-degeneracy, and this is the basis of the rooting procedure. In other words, if the symmetry/degeneracy were exact, it would be conceivable that one might reduce the degrees of freedom by another factor of $2^{d / 2}$ (analogous to Kogut and Susskind, but with different technical means). However, at finite lattice spacing there are always some configurations where the taste symmetry is not approximately restored (rightmost panel), and the issue concerning the "rooting trick" is basically the question whether these "bad guys" would retain non-zero measure in the continuum. 


\section{Problem: rooting versus locality}

The earliest warning regarding the legality of the "rooting trick" that I am aware of came in a paper by Marinari, Parisi and Rebbi in 1981: "On the lattice the action $S_{G}-\frac{1}{4} \operatorname{tr} \log \left(D_{\mathrm{st}, m}\right)$ will produce a violation of fundamental axioms, but we expect the violation to disappear in the continuum limit and then recover the theory with a single fermion" [11].

As mentioned in the introduction, the main problem is the potential loss of locality at finite lattice spacing. With any undoubled Dirac operator, e.g. $D=D_{\mathrm{W}}$, one has

$$
\left.Z\right|_{N_{f}}=\int D[U, \bar{\psi}, \psi] e^{-S_{G}[U]-\Sigma \int \bar{\psi} D \psi}=\int D[U] \operatorname{det}^{N_{f}}(D) e^{-S_{G}[U]}
$$

where the first sum extends over $N_{f}$ flavors, i.e. an integer power of the determinant emerges rather naturally with $N_{f}$ degenerate fermions. The question is whether one may take fractional powers if a formulation with doublers yields too many fermions in the continuum, specifically whether

$$
\left.Z\right|_{N_{f}}=\int D[U] \operatorname{det}^{N_{f} / 4}\left(D_{\text {st }, m}\right) e^{-S_{G}[U]}
$$

is a legal implementation if $N_{f}$ is not a multiple of 4 . The point is that it is not clear whether the resulting effective action $S_{\text {eff }}=S_{G}-\frac{N_{f}}{4} \operatorname{tr} \log \left(D_{\text {st }, m}\right)$ receives contributions from unphysical degrees of freedom which persist in the continuum. In principle, such a disaster might happen with any non-zero prefactor, and locality is thus a issue in the staggered approach for any $N_{f}>0$.

The reason why locality is so important is universality. We are all used to the notion that we do not need to worry about the details of the action used, since the continuum limit is a $2^{\text {nd }}$ order phase transition in the course of which all physical correlation lengths diverge and any knowledge about irrelevant pieces in the action is washed out. However, the standard theorems ensuring these nice properties build on the fundamental action being local (at finite lattice spacing) [12].

The soundness of the effective action would be guaranteed if one could "integrate in" a fermion in (3.2), i.e. if one could find a legal doubler-free "candidate" action $D_{\mathrm{ca}, m}$ with [13]

$$
\operatorname{det}^{1 / 4}\left(D_{\mathrm{st}, m}\right)=\int D[\bar{\psi}, \psi] e^{-\int \bar{\psi} D_{\mathrm{c}, m} \psi} .
$$

The attribute "legal" means that it satisfies the requirements

$$
\begin{aligned}
& \tilde{D}_{\mathrm{ca}}=\mathrm{i} \gamma_{\mu} p_{\mu}+O\left(p^{2}\right) \\
& \left\|D_{\mathrm{ca}}(x, y)\right\|<C e^{-v|x-y| / a} \text { with } C, v \text { independent of } U \\
& \left\|\delta D_{\mathrm{ca}}(x, y, U) / \delta U(z)\right\|<C e^{-v \min (|x-z|,|y-z|) / a} \text { (ditto) }
\end{aligned}
$$

where $(3.5,3.6)$ are designed to ensure locality in the space of couplings and in the gauge field, and "doubler-free" is formalized by demanding that $\tilde{D}_{\text {ca }}(p)$ is invertible at all non-zero momenta (mod $2 \pi / a$ ). Of course, one might allow for cut-off effects in (3.3), i.e. one might relax it to

$$
\operatorname{det}\left(D_{\mathrm{ca}, m}\right) \stackrel{a \downarrow 0}{\longrightarrow} \text { const } \cdot \operatorname{det}^{1 / 4}\left(D_{\mathrm{st}, m}\right)
$$

but the problem will be to prove that the difference is really just a cut-off effect. 


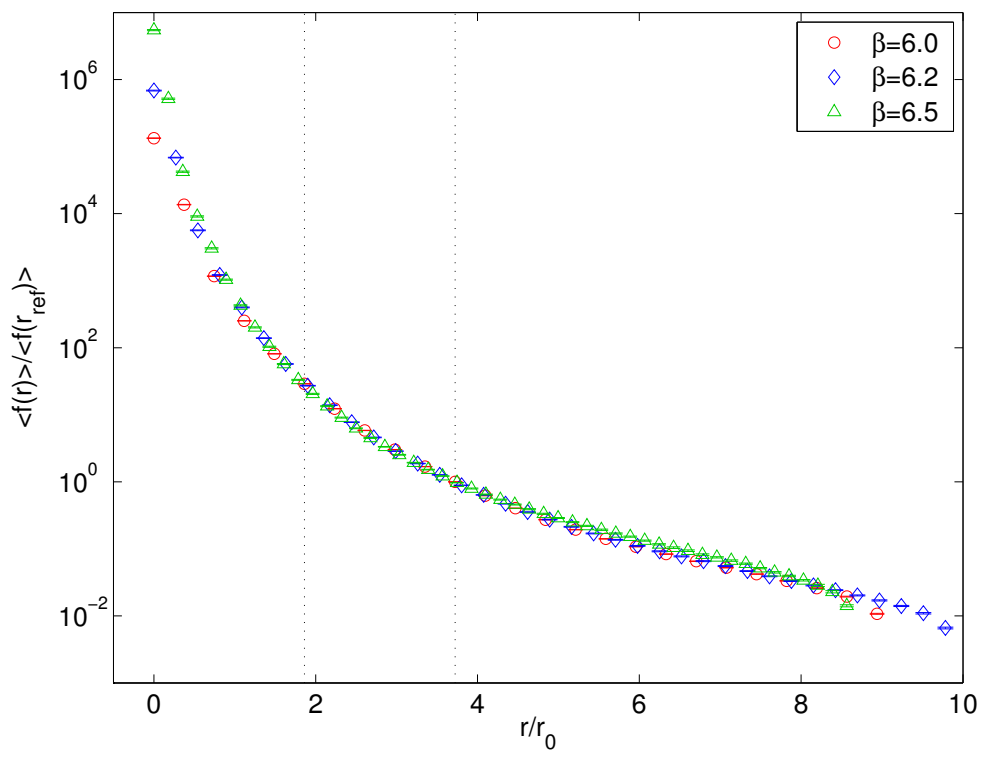

Figure 2: Localization function $f(r)$ in physical units versus $r / r_{0}$ for the "candidate" action of [15]. The local logarithmic derivative (the effective $r_{\text {loc }}$ ) scales and the operator is thus non-local. Figure from [15].

What might be a promising "candidate" action? Sometimes it is easier and more instructive to discuss first what is not a useful strategy. The staggered "rooting problem" is of this type, since it is evident that simply taking the fourth root of $D_{\text {st }, m}$ is not a viable option. Such an operator would have the right determinant, but it would violate other requirements, in particular $D_{\mathrm{ca}}=D_{\mathrm{st}}^{1 / 4}$ with $D_{\text {st }}$ the massless staggered operator would fail to obey (3.4).

That the technique [14] is available to decide whether a potential/proposed "candidate" action obeys the locality bound (3.5) has bee shown in [15]. These authors consider a slightly different "candidate" operator, $D_{\mathrm{ca}, m}=\left(D_{\mathrm{st}, m}^{\dagger} D_{\mathrm{st}, m}\right)_{\mathrm{ee}}^{1 / 2}=\left(-D_{\mathrm{st}}^{2}+m^{2}\right)_{\mathrm{ee}}^{1 / 2}>0$, and measure the localization function $f(r)=\sup \left\{\|\psi(x)\|_{2}|| \mid x-y \|_{1}=r\right\}$ where $\psi(x)=\sum D(x, z) \eta(z)$ with $\eta$ a normalized random vector at $y$. For a local $D$ one has $f(r) \propto e^{-r / r_{\mathrm{loc}}}$, ideally with $r_{\mathrm{loc}} \propto a$, but any $a$-dependence with $\xi_{\text {phys }} / r_{\text {loc }} \rightarrow \infty$ is fine. Employing matched lattices they find that $f\left(r / r_{0}\right)$ scales (and there is no convincing exponential fall-off pattern, see Fig. 2), i.e. the operator is non-local. Thus, apart from not qualifying as a fermion action since its spectrum lies on the real axis, this "candidate" operator may also be ruled out for more sophisticated reasons. Using an improved/filtered staggered action in the above-mentioned construct does not alter the conclusion [16], which is hardly surprising, since in the previous work the continuum limit has already been taken.

Still, the question remains: is there any "candidate" operator satisfying all requirements ? Note that finding a legal 1-flavor $D_{\text {ca, } m}$ is sufficient to show that staggered fermions yield an acceptable effective action. The continuum limit might be OK even if this is not the case, but in such an event a proof that the approach is safe would - almost for sure - require truly novel concepts.

\section{Free case: four constructions}

Given the importance of the issue whether a "candidate" action exists that satisfies all relevant criteria, it is encouraging to see that at least in the free theory the answer is positive and the task to 
construct such an operator has been completed. Interestingly, the solution is non-unique.

In [17] Adams starts from the observation that the massive staggered operator in the taste basis (2.3) on the blocked lattice may be used to build the combination

$$
D_{\mathrm{st}, m}^{\dagger} D_{\mathrm{st}, m}=\left[-\nabla^{2}+\frac{b^{2}}{4} \triangle^{2}+m^{2}\right]\left(I_{4} \otimes I_{4}\right)
$$

which is simultaneously diagonal in spinor $\otimes$ taste. Analogously, a free generalized Wilson operator $D_{\text {ca }, m}=\nabla_{\mu} \gamma_{\mu}+\frac{b}{2} W+m$ on the blocked lattice yields

$$
D_{\mathrm{ca}, m}^{\dagger} D_{\mathrm{ca}, m}=\left[-\nabla^{2}+\left(\frac{b}{2} W+m\right)^{2}\right]\left(I_{4} \otimes 1\right)
$$

without taste structure. With $\operatorname{det}\left(D_{\mathrm{st}, m}^{\dagger}\right)=\operatorname{det}\left(D_{\mathrm{st}, m}\right)$ and $\operatorname{det}\left(D_{\mathrm{W}, m}^{\dagger}\right)=\operatorname{det}\left(D_{\mathrm{W}, m}\right)$ it follows that $D_{\text {ca, } m}$ would trivially have the right determinant, if the two square brackets in (4.1) and (4.2) agree:

$$
\frac{b^{2}}{4} \triangle^{2}+m^{2}=\left(\frac{b}{2} W+m\right)^{2} \Longrightarrow \operatorname{det}^{1 / 4}\left(D_{\mathrm{st}, m}\right)=\operatorname{det}\left(D_{\mathrm{ca}, m}\right)
$$

Hence, a natural "candidate" operator in the free theory is $D_{\text {ca, } m}=\nabla_{\mu} \gamma_{\mu}+\sqrt{\frac{b^{2}}{4} \triangle^{2}+m^{2}}$ which lives on the blocked lattice. The less trivial part is to verify its locality. Using a technique developed by Fujikawa and Ishibashi he shows that the couplings fall off exponentially with $r_{\mathrm{loc}}=\sqrt{8 a / m} \stackrel{a \downarrow 0}{\longrightarrow} 0$ and this means that for $m>0$ the operator is local.

In [18] Maresca and Peardon investigate the massless case. They start from a "candidate" operator $D_{\text {ca }}=\mathrm{i} \gamma_{\mu} P_{\mu}+Q$ on the blocked lattice with local (i.e. not ultra-local) $P_{\mu}$ and $Q$. Again, $\operatorname{det}\left(D_{\text {ca }}\right)=\operatorname{det}^{1 / N_{t}}\left(D_{\text {st }}\right)$ is trivially realized if they can ensure that $D_{\mathrm{ca}}^{\dagger} D_{\mathrm{ca}} \otimes I_{N_{t}}^{\mathrm{taste}}=D_{\mathrm{st}}^{\dagger} D_{\mathrm{st}}$. To that aim they consider the ansatz $P_{\mu}=\sum_{r \geq 0} \sum_{|d| \leq r} \omega_{p, \mu}^{r, d}(x, y)$ and ditto for $Q$ in terms of a tower of operators with ever larger footprint, i.e. $\omega_{p, \mu}^{r, d}, \omega_{q}^{r, d}$ have range $r$. Ensuring the main criterion does not uniquely pin down all the coefficients, and they use this freedom to optimize $D_{\text {ca }}$ for locality. This optimization is performed numerically, both in $2 \mathrm{D}\left(N_{t}=2\right)$ and in $4 \mathrm{D}\left(N_{t}=4\right)$. With this strategy they make sure that $D_{\text {ca }}$ has the right determinant [my understanding: up to the exact (non-topological) zero-modes], and it turns out that the resulting operator is indeed exponentially localized. Presumably triggered by the spectrum of their "candidate" operator in 2D (see Fig. 3) they went on to ask whether they could make it obey the Ginsparg Wilson relation [19]. Indeed, considering only $Q$ of the form $Q=-\triangle R$ with a local $R$ makes the "candidate" operator satisfy

$$
\left\{D_{\mathrm{ca}}, \gamma_{5}\right\}=D_{\mathrm{ca}} 2 R \gamma_{5} D_{\mathrm{ca}}
$$

but the price to pay is that the localization range increases.

In [20] Shamir uses the renormalization group (RG) framework to deal with the free case. The general idea is that even in the interacting theory performing $n$ blocking steps (for simplicity: $n$ coarse grainings by a factor 2) creates a "two cut-off" situation where the physics accessible on the coarse lattice is given by the final cut-off $a^{-1} / 2^{n}$, but the taste splitting is still given by the original $a^{-1}$. Thus, starting from an ever finer lattice and applying more blocking steps is a way to improve the taste symmetry (cf. talk by F. Maresca [21]). Infinitely many steps render it exact, i.e. $D_{n} \rightarrow D_{\infty} \otimes I_{4}$, while $D_{\text {ca }}=D_{n}$ after $n$ steps satisfies $\operatorname{det}^{1 / 4}\left(D_{\text {st }}\right)=\operatorname{det}\left(D_{\text {ca }}\right) \operatorname{det}^{1 / 4}(T)$ where $T$ 


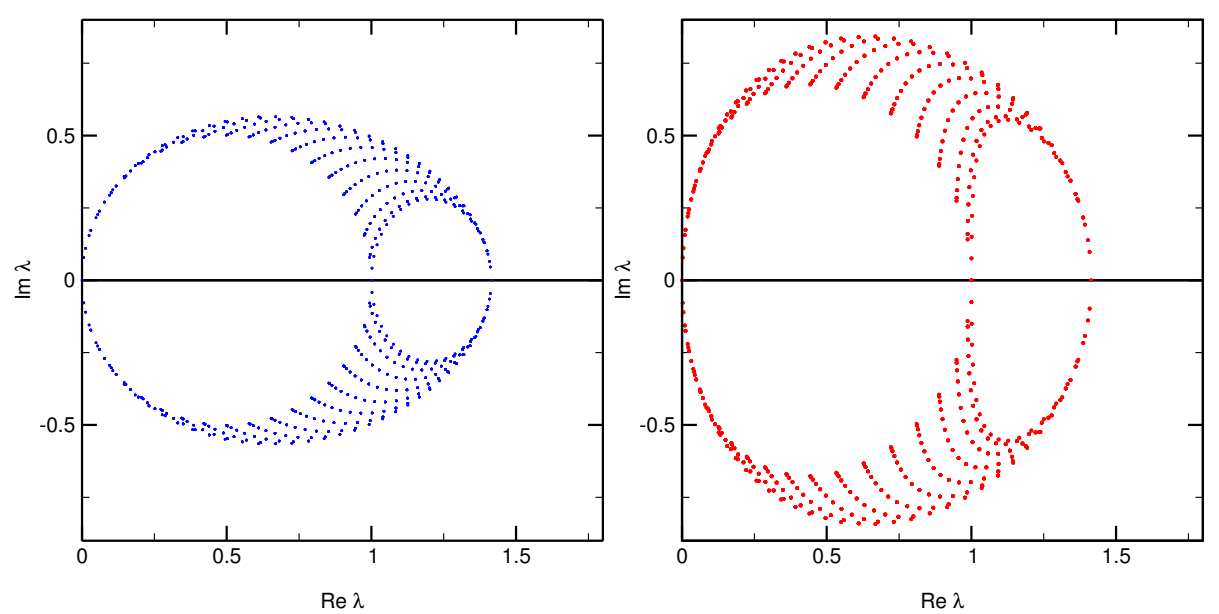

Figure 3: Eigenvalue spectra of two "candidate" operators $D_{\text {ca }}$ on the blocked lattice in $2 \mathrm{D}$ which reproduce $\operatorname{det}^{1 / 2}\left(D_{\text {st }}\right)$ of the massless staggered action in the free theory. On the left $D_{\text {ca }}$ is exclusively optimized for locality, on the right the (standard) Ginsparg Wilson relation is imposed as a constraint. Figure from [18].

contains only (original) cut-off excitations and should maintain the Symanzik class, i.e. $\operatorname{det}(T)=$ const $\cdot\left(1+O\left(a^{2}\right)\right)$. The massless staggered action on the original lattice satisfies $\left\{D_{0},\left(\gamma_{5} \otimes \tau_{5}\right)\right\}=0$. Interestingly, the action after $n$ blocking steps satisfies a generalized Ginsparg Wilson relation [20]

$$
\left\{D_{n},\left(\gamma_{5} \otimes \tau_{5}\right)\right\}=D_{n} \frac{2}{\alpha_{n}}\left(\gamma_{5} \otimes \tau_{5}\right) D_{n}
$$

where $\alpha_{n}$ is a parameter of the blocking procedure. If (4.5) were complemented with a $\left(\gamma_{5} \otimes \tau_{5}\right)$ hermiticity of $D_{n}$, one would easily obtain $D_{n}+D_{n}^{\dagger}=D_{n} \frac{2}{\alpha_{n}} D_{n}^{\dagger}$, i.e. that the spectrum of $D_{n}$ lies on a Ginsparg-Wilson circle. However, it is straightforward to derive spectral properties without such $\mathrm{a}\left(\gamma_{5} \otimes \tau_{5}\right)$-hermiticity. Start from the representation $D_{n}=\sum_{j} \lambda_{j} u_{j} v_{j}^{\dagger}$ in terms of bi-orthogonal R/Leigenvectors, i.e. $v_{i}^{\dagger} u_{k}=\delta_{i, k}$. Upon sandwiching the GW relation (4.5) with $v_{i}^{\dagger}$ and $u_{k}$, one obtains $\lambda_{i} v_{i}^{\dagger}(\ldots) u_{k}+v_{i}^{\dagger}(\ldots) u_{k} \lambda_{k}=\lambda_{i} v_{i}^{\dagger} \frac{2}{\alpha_{n}}(\ldots) u_{k} \lambda_{k}$ where ... stands for $\gamma_{5} \otimes \tau_{5}$. Thus, for an arbitrary pair $(i, k)$ of eigenmodes, one has $v_{i}^{\dagger}\left(\gamma_{5} \otimes \tau_{5}\right) u_{k}=0$ or $\lambda_{i}+\lambda_{k}-\frac{2}{\alpha_{n}} \lambda_{i} \lambda_{k}=0$. In particular for $i=k$ it follows that $v_{j}^{\dagger}\left(\gamma_{5} \otimes \tau_{5}\right) u_{j} \neq 0$ necessarily implies $2 \lambda_{j}-\frac{2}{\alpha_{n}} \lambda_{j}^{2}=0$. Hence the spectrum crosses the real axis at $\lambda_{j} \in\left\{0, \alpha_{n}\right\}$. The more general situation with a spectrum between two "Hasenfratz-Niedermayer circles" (tangent to the imaginary axis) is avoided, and the reason why is simply that Shamir uses a non-overlapping blocking kernel.

There are two more articles to be mentioned. In [22] Giedt gives an exploratory discussion of the interacting case, based on RG concepts. In [23] Neuberger uses a different setup; he recasts the question of the validity of the "rooting trick" into a local field theoretical framework in 6D.

\section{Interacting theory: $\operatorname{spec}\left(D_{\text {st }}\right)$ in $4 \mathrm{D}$}

Before discussing what can be learned from analyzing spectral properties of the staggered Dirac operator, let me recall the concept of filtering. What is meant is simply a change of the covariant derivative in the fermion action $[24,25,26,27,28,29]$ through a "fat link" recipe like

$$
U_{\mu}(x) \psi(x+\hat{\mu})-\psi(x) \quad \longrightarrow \quad U_{\mu}^{\mathrm{HYP}}(x) \psi(x+\hat{\mu})-\psi(x) .
$$



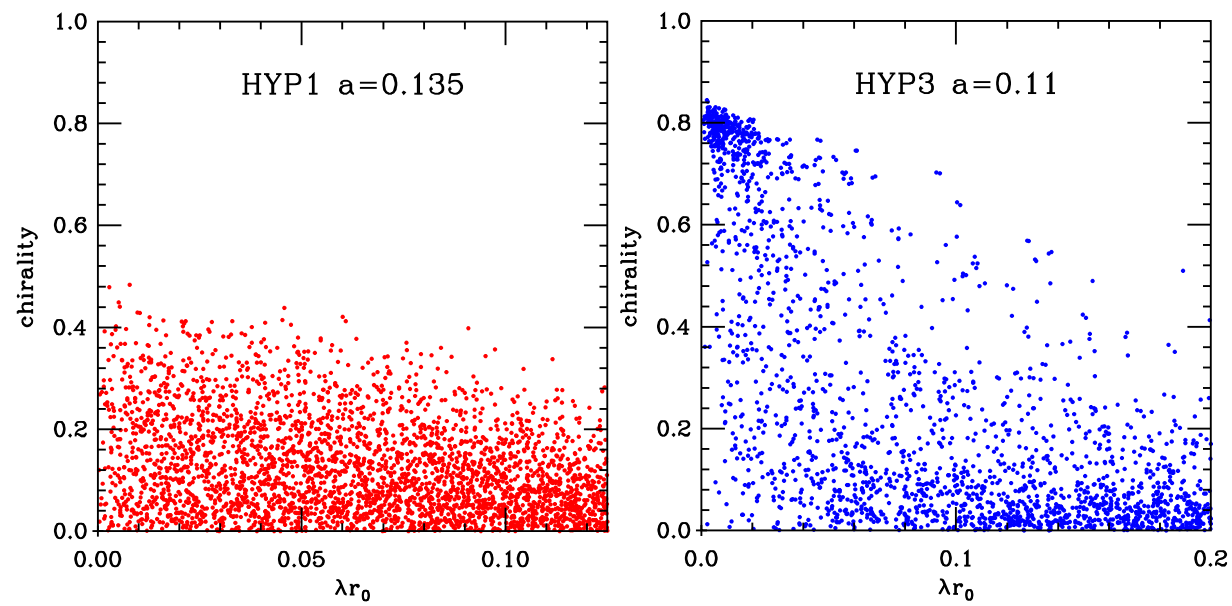

Figure 4: Chirality versus eigenvalue of staggered eigenmodes. On coarse lattices there is no distinctive signature, but on somewhat finer lattices rather aggressive filtering reveals continuum like features. Nearzero modes are predominantly chiral, non-zero modes are not. Figure from [33].

The idea is that such a modification alters/diminishes cut-off effects without changing the Symanzik class, i.e. a theory with $O\left(a^{2}\right)$ cut-off effects will retain this characteristics, but the extrapolation slope is changed (typically reduced). For staggered quarks the obvious design goal of a filtering prescription is to render $D_{\text {st }}$ "immune" against $p \sim \pi / a$ gluons, and the question is how efficient a given recipe is at ameliorating the taste symmetry. For Wilson fermions the additive mass renormalization gets reduced through the modification (5.1) and for related technical reasons even the overlap operator [30] benefits from such a filtering [31, 32].

An excellent starting point is found in a talk by A. Hasenfratz [33]. In the (formal) continuum $D \psi=\lambda \psi \Longleftrightarrow D \gamma_{5} \psi=-\lambda \gamma_{5} \psi$, thus non-zero modes come in complex conjugate pairs ( $\lambda$ is purely imaginary) and have zero chirality $\left(\psi^{\dagger} \gamma_{5} \psi=0\right.$ if $\lambda \neq 0$ ), while zero modes have non-vanishing chirality $\left(\zeta^{\dagger} \gamma_{5} \zeta= \pm 1\right.$ if $\left.D \zeta=0\right)$. On coarse lattices unfiltered and modestly filtered staggered quarks show no indication of such "continuum like" behavior (see left panel of Fig. 4), but with $a^{-1} \simeq 2 \mathrm{GeV}$ and some rather aggressive filtering the situation changes, on a qualitative level at least (see right panel of Fig. 4). The idea is that the same picture would emerge with the "thin link" action, too, if one could push to much smaller lattice spacings (say $a^{-1} \simeq 20 \mathrm{GeV}$ or so). At least it becomes evident that cut-off effects may completely mask the underlying continuum theory.

A more complete investigation has been carried out and presented last year by Follana et al. $[34,35]$. The difference is that they show results for individual configurations and compare several filtering recipes and gauge actions (at fixed lattice spacing). Furthermore, they determine the (gluonic) topological charge $q$ of their configurations. With lattice spacings $a^{-1} \simeq 2 \mathrm{GeV}$ only the combination of an improved gauge action and a highly filtered $D_{\text {st }}$ allows to see a separation into near-zero modes with non-vanishing chirality and non-zero modes without chirality (cf. Fig. 5). Furthermore, the number of near-zero modes is $4|q|$ (i.e. $2|q|$ on either side of the spectrum), and this means that staggered quarks satisfy an approximate index theorem.

The next step has been taken in a paper together with Christian Hoelbling and Urs Wenger where two new features come in [31]. The first one is that we compare to the overlap spectrum on the same configuration. Apart from finding $4|q|$ staggered near-zero modes on typical backgrounds 

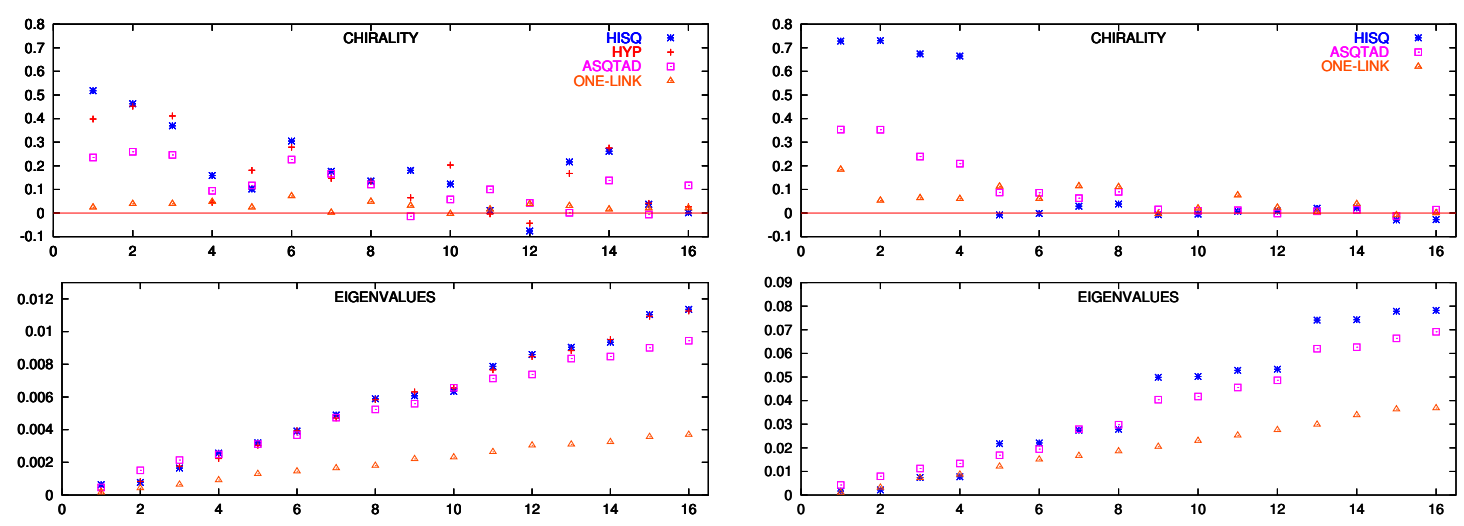

Figure 5: Chirality and eigenvalue versus "serial number" of the lowest few staggered eigenmodes of the positive staggered half-spectrum. The left panel is for Wilson glue, the right one for the Symanzik action, with $a \simeq 0.1 \mathrm{fm}$ in either case. The staggered action with "HISQ" filtering sees $|q|=2$. Figure from [34].
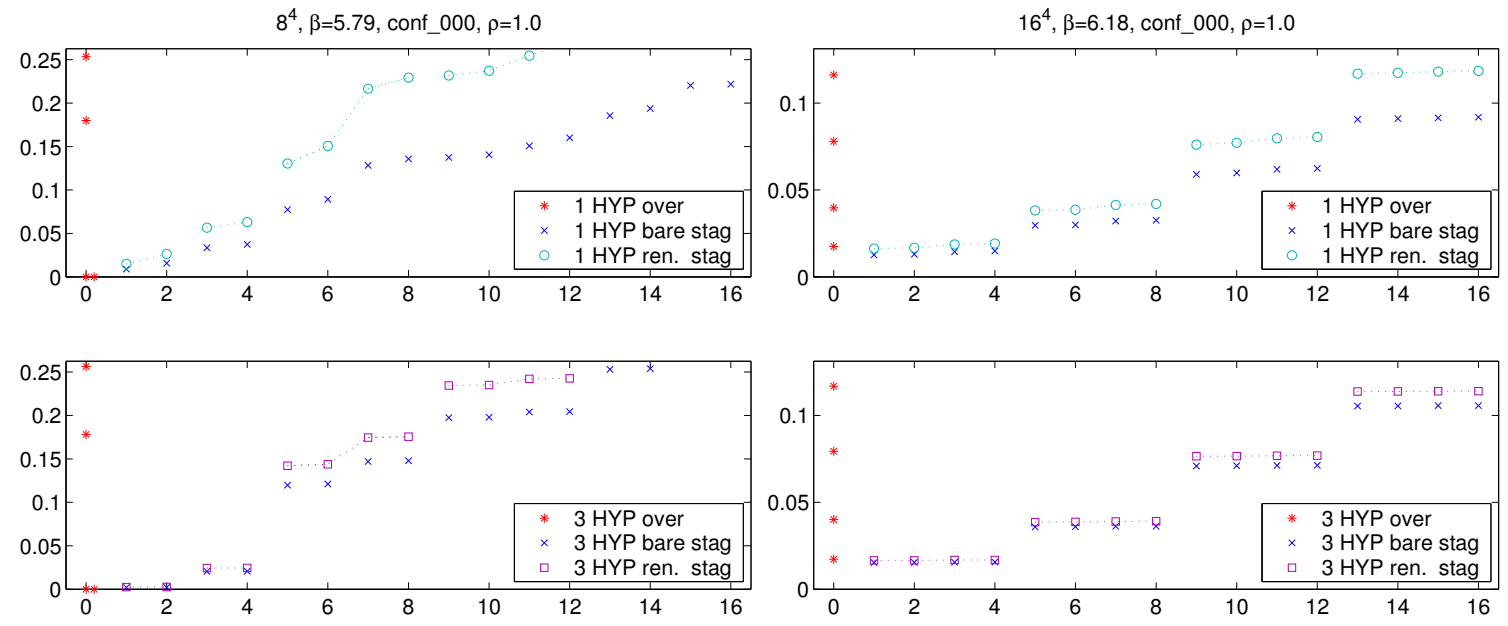

Figure 6: Same as the lower part of Fig. 5, but with two different lattice resolutions (keeping the physical volume fixed) and with overlap eigenvalues on the same configuration for comparison. After rescaling with $Z_{S}^{\text {st }} / Z_{S}^{\text {ov }}$ one sees a quantitative single-overlap to staggered-quartet agreement. Figure from [31].

with overlap charge $q$ we show that after rescaling with the relative $Z_{S}$-factor one finds a quantitative agreement of staggered quartets with the (non-degenerate) overlap eigenvalues (cf. Fig 6). The second new ingredient is that we compare several matched lattices (with fixed physical volume) and investigate how the intra-quartet splitting decreases with a finer resolution (see below).
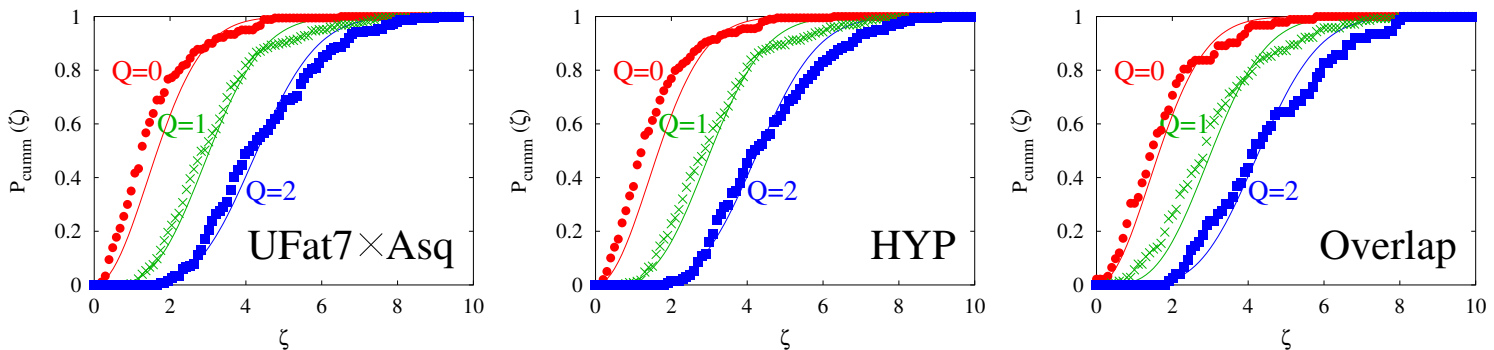

Figure 7: Cumulative eigenvalue distribution for two filtered $D_{\text {st }}$ and the "thin-link" $D_{\text {ov }}$. Figure from [36]. 

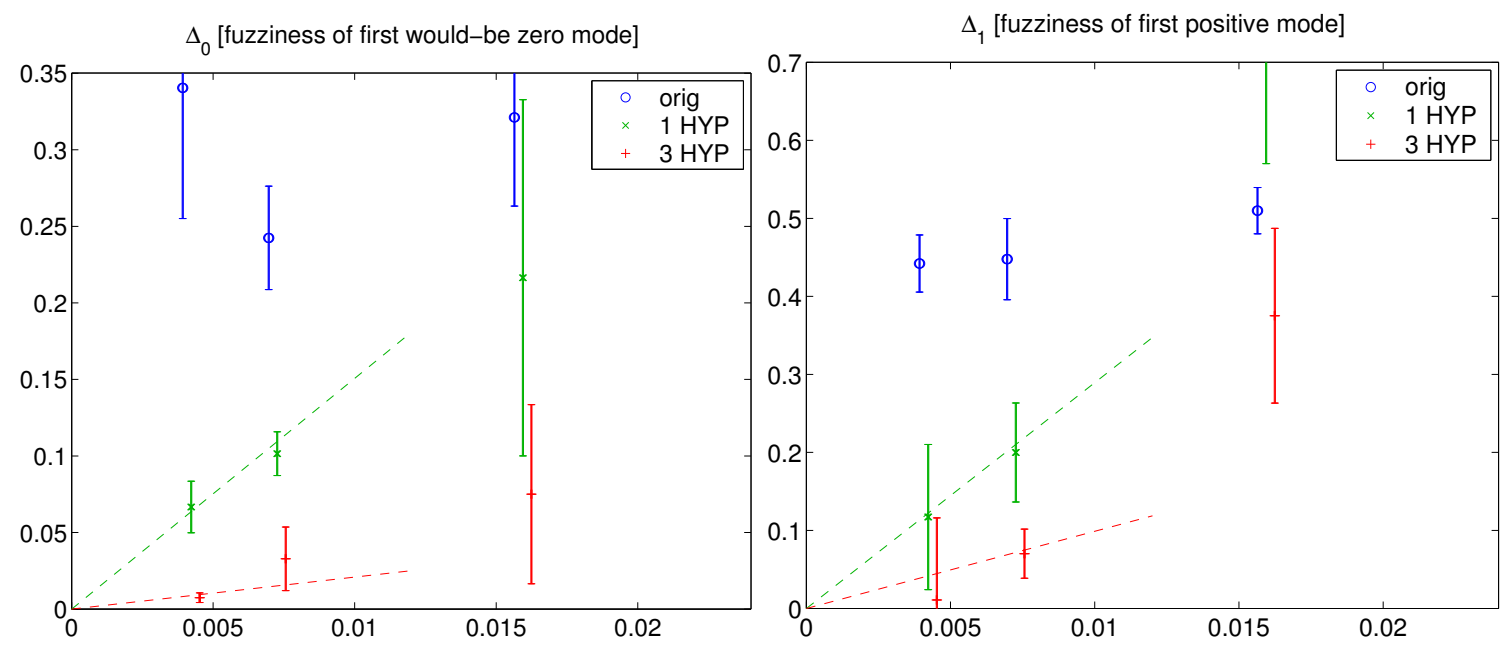

Figure 8: Staggered near-zero eigenvalue (left) and intra-quartet splitting (right) versus the lattice spacing squared [everything in physical units]. With filtering one might be in the scaling window. Figure from [31].
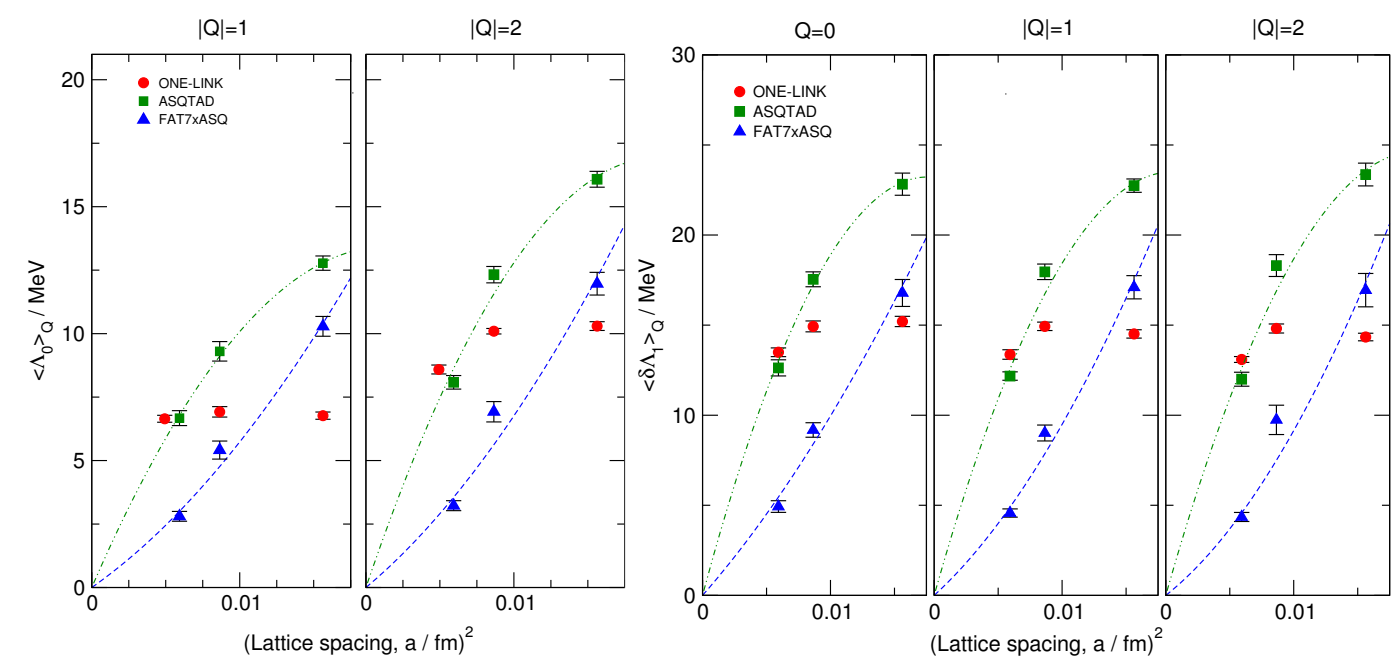

Figure 9: Same as Fig. 8, but separated according to $|q|$ and with much better statistics. Figure from [37].

For quite some time it was believed that staggered quarks are insensitive to topology and would never reproduce the sectoral random matrix theory (RMT) predictions for the distribution/ratio of the lowest eigenvalues. Given the correspondence to the overlap spectrum it is clear that this cannot be true. In [36] it is shown that on sufficiently fine lattices and with filtering the agreement is just as good as with the overlap action (see Fig. 7). In [34] it was already shown that the ratio of low-lying eigenvalues [i.e. $\left\langle\lambda_{i}\right\rangle /\left\langle\lambda_{j}\right\rangle$ with $\lambda_{i}$ denoting a staggered quartet mean] satisfies the RMT prediction, up to small violations which might be finite volume effects or remnant discretization errors.

The last point to be covered is the evidence that the staggered taste symmetry violation might indeed be an $O\left(a^{2}\right)$ cut-off effect. A first attempt was made in [31] where the taste splitting is defined through the "spread" of a staggered quartet that is to mimic a (single) overlap mode. Plotting the splitting versus $a^{2}$, everything seems consistent with the hypothesis that it might disappear in proportion to $a^{2}$ [with filtering, without one is certainly not in the scaling regime], see Fig. 8 . In [37] these findings have been corroborated with much better statistics (see Fig. 9) and in [21] a 
more physical definition of the taste splitting has been found to lead to the same overall picture.

In summary, investigations in 4D have so far revealed evidence in favor of the "rooting trick". The problem is just that even the most convincing numerical evidence does not amount to a proof.

\section{Interacting theory: $\chi_{\text {sca }}, \chi_{\text {top }}$ in $2 D$}

The main reason for working in 2D is that for a large number of observables a rather impressive statistical precision can be reached. Hence, if there is a problem with the staggered approach, one might think that it is most likely to be detected in such a setup.

In $2 \mathrm{D}$ a square root of the determinant is used for an odd number of dynamical fermions, since the staggered action yields 2 fermions in the continuum. Results below are in the (generalized) Schwinger model [38], i.e. QED in 2D with $N_{f}$ massive fermions. Note that the fundamental charge $g$ has the dimension of a mass and does not run. Therefore, $g$ may be used to set the scale, and in this case the relation $\beta=1 /(a g)^{2}$ holds exact. For $N_{f}=1$ there is the analytic result [38]

$$
\lim _{m \downarrow 0} \frac{\langle\bar{\psi} \psi\rangle}{g}=\frac{e^{\gamma}}{2 \pi^{3 / 2}}=0.1599 \ldots
$$

which is due to the axial anomaly and does not signal spontaneous symmetry breaking.

The challenge is to see whether one may directly reproduce (6.1) with staggered fermions. To this aim we sample quenched, compute all eigenvalues (both of $D_{\text {st }}$ and $D_{\text {ov }}$ to have a cross check, and in either case with several filterings) and determine the observable in the analysis program via

$$
\begin{array}{cl}
\frac{\chi_{\text {sca }}^{\mathrm{ov}}}{g}=\frac{\sqrt{\beta}}{V} \frac{\left\langle\operatorname{det}^{N_{f}}\left(D_{m}^{\mathrm{ov}}\right) \sum \frac{1}{\hat{\lambda}+m}\right\rangle}{\left\langle\operatorname{det}^{N_{f}}\left(D_{m}^{\mathrm{ov}}\right)\right\rangle}, & \frac{\chi_{\text {sca }}^{\mathrm{st}}}{g}=\frac{\sqrt{\beta}}{2 V} \frac{\left\langle\operatorname{det}^{N_{f} / 2}\left(D_{m}^{\mathrm{st}}\right) \sum \frac{1}{\lambda+m}\right\rangle}{\left\langle\operatorname{det}^{N_{f} / 2}\left(D_{m}^{\mathrm{st}}\right)\right\rangle} \\
\operatorname{det}\left(D_{m}^{\mathrm{ov}}\right)=\prod\left(\left(1-\frac{m}{2}\right) \lambda+m\right), & \operatorname{det}\left(D_{m}^{\mathrm{st}}\right)=\prod(\lambda+m)
\end{array}
$$

where $\hat{\lambda}=(1 / \lambda-1 / 2)^{-1}$ denotes the stereographically mapped overlap eigenvalues. The prefactor $\frac{1}{2}$ in the staggered expression is designed to compensate for the 2 tastes contributing; this is the valence counterpart to the "rooting trick" in the sea sector. The bare condensates (6.2) have a logarithmic divergence of type $m / g \cdot \log (m a)$, i.e. the free case needs to be subtracted, but there is no multiplicative renormalization. For technical details see [39, 40]. All plots below will have $m_{\text {sea }}=m_{\text {val }}$, i.e. at least with $D=D_{\text {ov }}$ the theory is exactly unitary (at finite lattice spacing).

Given this setup it is clear that we can draw "continuous" $1 \sigma$ (statistical) error bands for the subtracted condensate $\chi_{\text {sca }}^{\prime} / g$ as a function of $m / g$. The volume is chosen such that the Compton wavelength of the lightest degree of freedom in the chiral limit for $N_{f}=1$ fits $4-5$ times into the box. The first observation in Fig. 10 is that our overlap data satisfy the continuum expectation

$$
\chi_{\text {sca }}^{\prime} / g \propto \begin{cases}g / m & \left(N_{f}=0\right) \\ \text { const } & \left(N_{f}=1\right) \\ m / g & \left(N_{f}=2\right)\end{cases}
$$

at least on a qualitative level. Moreover, the overlap results with and without filtering look almost identical. And finally, in the chiral limit the 1-flavor result is consistent with the exact value (6.1). With staggered fermions the picture looks rather different. The most obvious problem is that in 

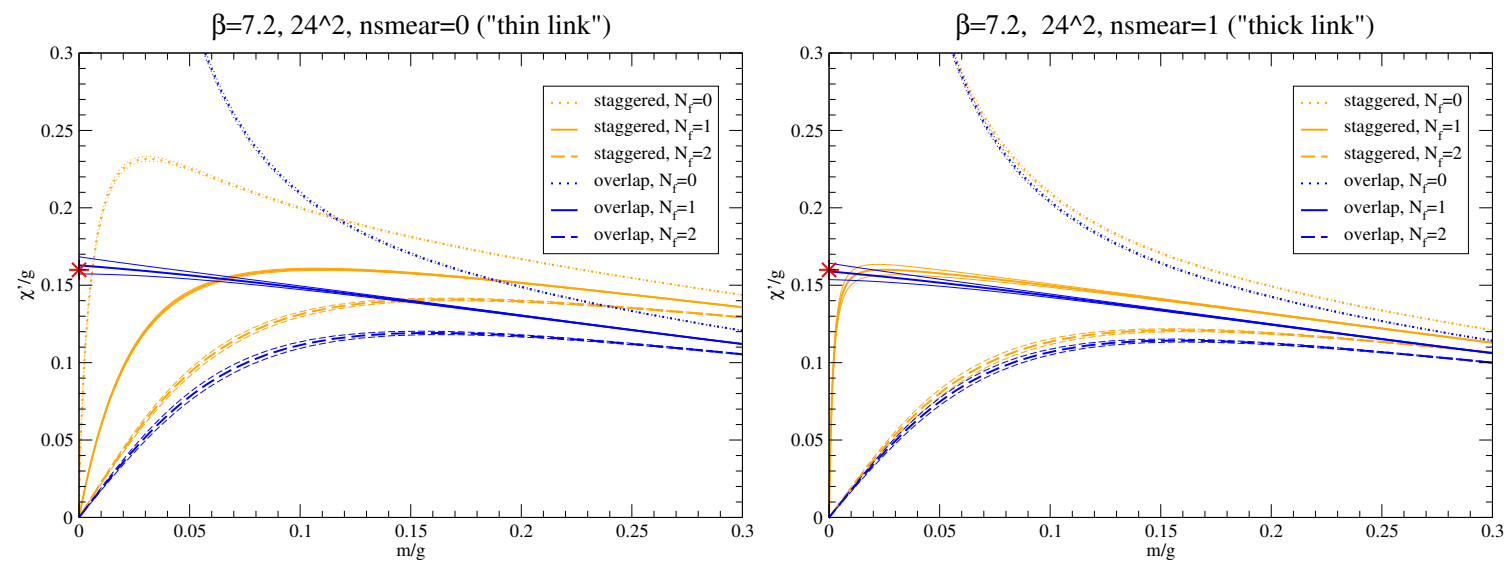

Figure 10: Scalar condensate versus quark mass (everything in physical units) in the Schwinger model, with $D_{\text {st }}$ and $D_{\text {ov }}$, with $N_{f}=0,1,2$, without (left) or with filtering (right). Figure (with modification) from [39].

the chiral limit these condensates tend to zero for all $N_{f}$, in marked contrast to (6.4). However, at the coupling used a single filtering step suffices to dramatically improve the situation - for nottoo-small quark masses at least. Still one has $\lim _{m \rightarrow 0} \chi_{\text {sca }}^{\prime \text { st }} / g=0$ at finite lattice spacing (even for $N_{f}=0$ where the slope is so steep that already the first pixel is off scale), but the point where this happens has been shifted to the left. This is in line with our previous observation that the splitting within staggered eigenvalue doublets gets dramatically reduced through filtering (cf. Fig. 1 and Ref. [39]). However, there is a large numerical factor relating the intra-taste eigenvalue splitting and the breakdown mass, and this ought to be understood before one claims a relationship. The main question that emerges from the r.h.s. of Fig. 10 is whether one can reproduce (6.1) with staggered fermions if one first takes the continuum limit (at finite $\mathrm{m} / \mathrm{g}$ ), pushing to the chiral limit in a second step. It turns out that this is indeed the case, viz.

$$
\begin{array}{ll}
\lim _{a \rightarrow 0} \lim _{m \rightarrow 0} \frac{\chi_{\mathrm{sca}}^{\text {ov }}\left(m / g, a^{2}\right)}{g}=\frac{e^{\gamma}}{2 \pi^{3 / 2}}, & \lim _{a \rightarrow 0} \lim _{m \rightarrow 0} \frac{\chi_{\mathrm{sca}}^{\prime \text { st }}\left(m / g, a^{2}\right)}{g}=0 \\
\lim _{m \rightarrow 0} \lim _{a \rightarrow 0} \frac{\chi_{\text {sca }}^{\prime \text { ov }}\left(m / g, a^{2}\right)}{g}=\frac{e^{\gamma}}{2 \pi^{3 / 2}}, & \lim _{m \rightarrow 0} \lim _{a \rightarrow 0} \frac{\chi_{\text {sca }}^{\text {st }}\left(m / g, a^{2}\right)}{g}=\frac{e^{\gamma}}{2 \pi^{3 / 2}}
\end{array}
$$

where every identity except the one with the exact zero is to be read as "our data are consistent with the hypothesis that ... " [40]. Thus there is a non-commutativity phenomenon specific to the staggered action. In other words, for this theory the staggered action is not in the right universality class, if one implements $\chi_{\text {sca }} / g$ through (6.2) and insists on a particular "bad" order of limits. Perhaps more surprising to staggered skeptics is that the "good" order of limits (first $a \rightarrow 0$, then $m \rightarrow 0$ ) brings it to the right universality class. This means that staggered quarks - with the help of an extra guiding principle - do perceive the chiral anomaly [40].

What makes staggered quarks fail, in this specific case, with the "bad" order of limits ? We did two experiments. Already in [39] we asked what happens if one combines staggered sea with overlap valence quarks or vice versa (BTW, [39] contains the first published "hybrid action" test). In such a setup one has the "taste reduction" problem only in one of the two sectors. We restrict ourselves to $m_{\mathrm{sea}}=m_{\mathrm{val}}$. As shown in Fig. 11 the results with overlap valence quarks on staggered sea look similar to the genuine staggered data shown before. Again, the curves tend to zero below 

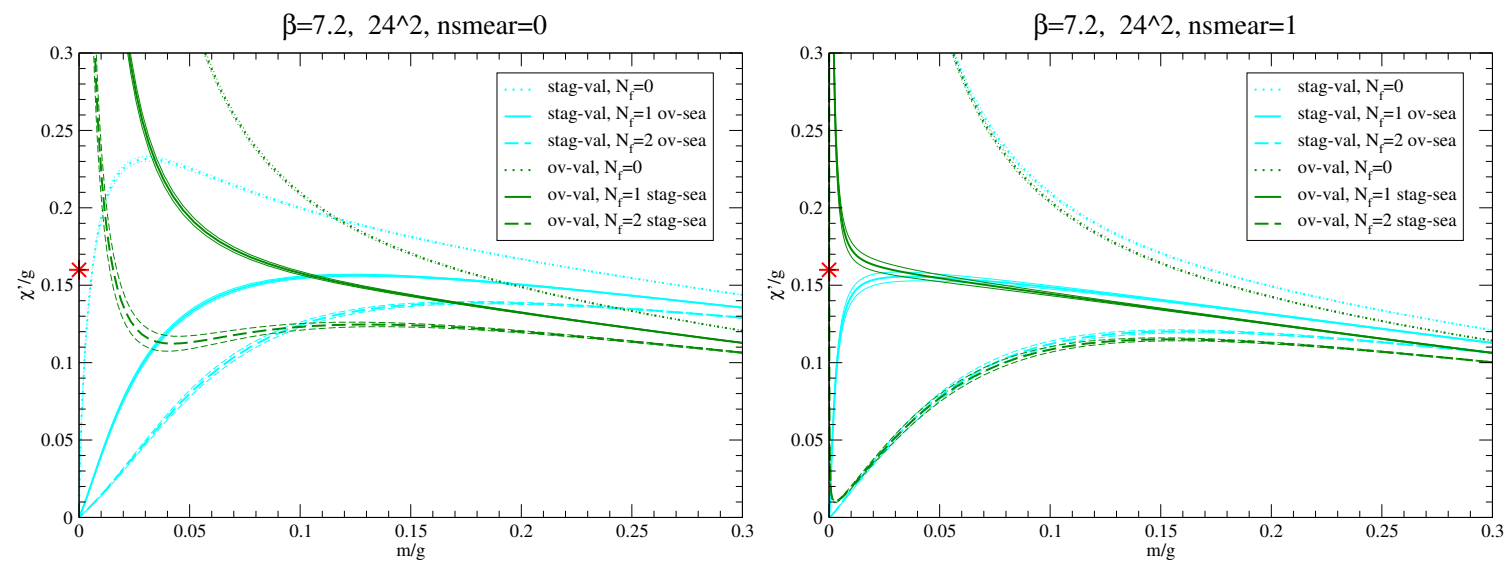

Figure 11: Same as Fig. 10, but for the formulations overlap valence on staggered sea (dark) and staggered valence on overlap sea (light), without (left) and with filtering (right). Figure (with modification) from [39].

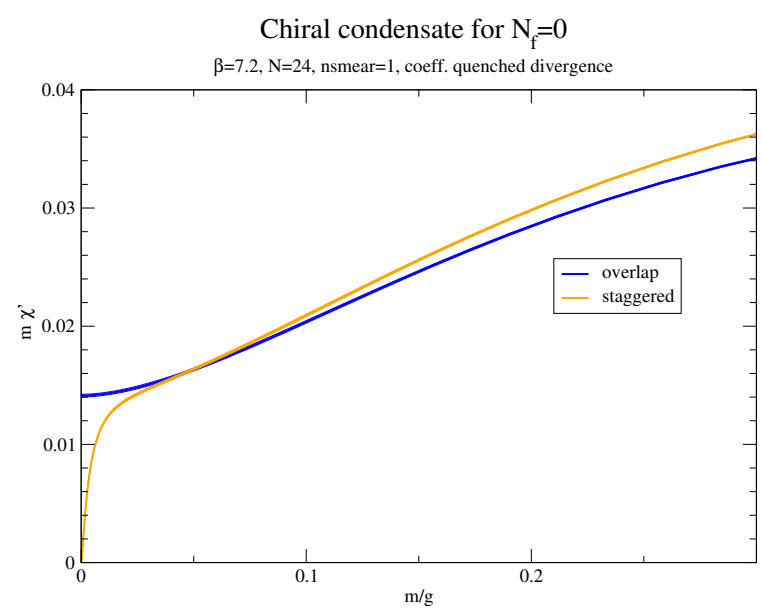

Figure 12: Attempt to "zoom in" on the coefficient of the divergence in the $N_{f}=0$ case. Overlap fermions yield the right result, regardless whether $m \rightarrow 0$ or $a \rightarrow 0$ is taken first. With staggered fermions another non-commutativity shows up which does not involve a rooted determinant. Figure previously unpublished.

some "critical" quark mass, and the latter happens to be more pronounced (and smaller) with filtering than without. An interesting observation concerns the reverse combination, staggered valence quarks on overlap sea (which in 4D would be a crazy thing to do). Here, the curves blow up at the same "critical" mass. At least in this example the notion of exact chiral symmetry in either the valence or the sea sector alone does not enable one to go closer to the chiral limit (at fixed $a$ ) than with the generic "staggered on staggered" combination. This is potentially bad news for modern attempts to evaluate overlap/domain-wall valence quarks on "cheap" staggered ensembles (see $[41,42]$ for a discussion of specific issues and $[43,44]$ for the effective theory). For our problem the bottom line is that the failure of the staggered action in the 1-flavor theory with the "bad" order of limits cannot be attributed to the sea or valence sector alone.

The second experiment is the result of a discussion with Claude Bernard. To find out whether the non-commutativity $(6.5,6.6)$ is causally connected to the "rooting trick" in the 1-flavor theory with staggered fermions, we checked whether one can compute the coefficient of the quenched divergence in (6.4) with staggered fermions. In Fig. 12 the product $m \chi_{\text {sca }}^{\prime \text { st }}$ iov is plotted versus $m / g$. 

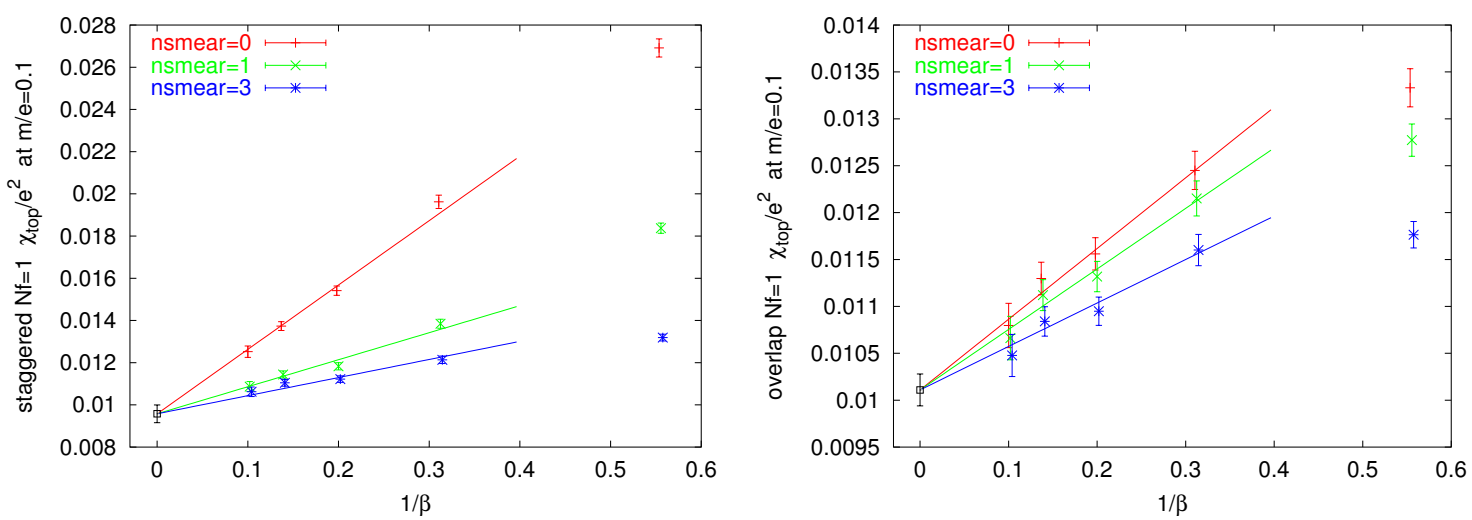

Figure 13: Topological susceptibility $\chi_{\mathrm{top}} / g^{2}$ in the 1 -flavor theory with $\operatorname{det}^{1 / 2}\left(D_{\text {st }, m}\right)$ (left) or $\operatorname{det}\left(D_{\text {ov }, m}\right)$ (right) at fixed $m / g=0.1$, plotted versus $(a g)^{2}$. The continuum limit seems to be universal. Figure from [40].

With overlap quarks the order of the chiral and the continuum limit is irrelevant. With staggered quarks taking $m \rightarrow 0$ first yields a vanishing result, hence taking $a \rightarrow 0$ in the second step leads to an exact zero. However, the result is different if one reverses the order of limits. With $a \rightarrow 0$ the "dip" in Fig. 12 gets filled in for every finite $m / g$. Therefore, taking $m \rightarrow 0$ in the second step one ends up with a finite result (which is conjectured to be identical to the coefficient obtained from overlap fermions in the combined chiral and continuum limit).

At the moment it is not clear what the precise implication of any of these experiments is for the case of interest (QCD with $N_{f}=2+1$ quarks at their physical masses). Evidently, the chiral limit at finite lattice spacing seems to be troublesome for staggered fermions. This has already been seen by Smit and Vink in Ref. [45] where they determine (mass dependent) renormalization factors for the topological charge as seen by the massive $D_{\text {st }, m}$. These factors tend to diverge for $m \rightarrow 0$. Our contribution was just to promote this into troubles with a real physical observable. In any case the bottom line is that the non-commutativity $(6.5,6.6)$ is not tied to the "rooting trick" per se but rather reflects a general mismatch between the sea and valence sector. This fits the view by Bernard who observes that similar non-commutativities show up in the effective theory for staggered fermions in 4D with partial quenching, i.e. if the sea and valence quarks have different masses [46].

Finally, I should add that the non-commutativity $(6.5,6.6)$ is more the exception than the rule. We checked a variety of other observables in the Schwinger model and did not find anything similar. To give an idea Fig. 13 shows our data for the $N_{f}=1$ topological susceptibility $\chi_{\text {top }} / g^{2}$ at fixed physical quark mass. Having data with three filtering levels and with several couplings in the Symanzik scaling regime allows for a fairly safe extrapolation to the continuum. The results with the two discretizations seem consistent, and also the exact continuum result $\lim _{m \rightarrow 0} \chi_{\text {top }} / g^{2}=0$ is reproduced with staggered fermions, regardless whether $m \rightarrow 0$ or $a \rightarrow 0$ is taken first [40].

\section{Correlation of $\operatorname{det}^{1 / N_{t}}\left(D_{\mathrm{st}, m}\right)$ and $\operatorname{det}\left(D_{\mathrm{ov}, m}\right)$}

We have seen, both in $2 \mathrm{D}$ and in $4 \mathrm{D}$, that $N_{t}$-fold nearly degenerate staggered eigenvalues manage to "mimic" a (single) overlap eigenvalue, e.g. $\lambda_{k}^{\text {ov }} \simeq \gamma_{k}^{\text {st }}$ with $\gamma_{k}^{\text {st }}=\mathrm{i}\left(-\lambda_{2 k-1}^{\text {st }} \lambda_{2 k}^{\text {st }}\right)^{1 / 2}$ in $2 \mathrm{D}$. Obviously, such a relation cannot extend to the entire spectrum (they are rather different in the UV, and the number of modes does not match), but the relevant question is whether it would extend to 


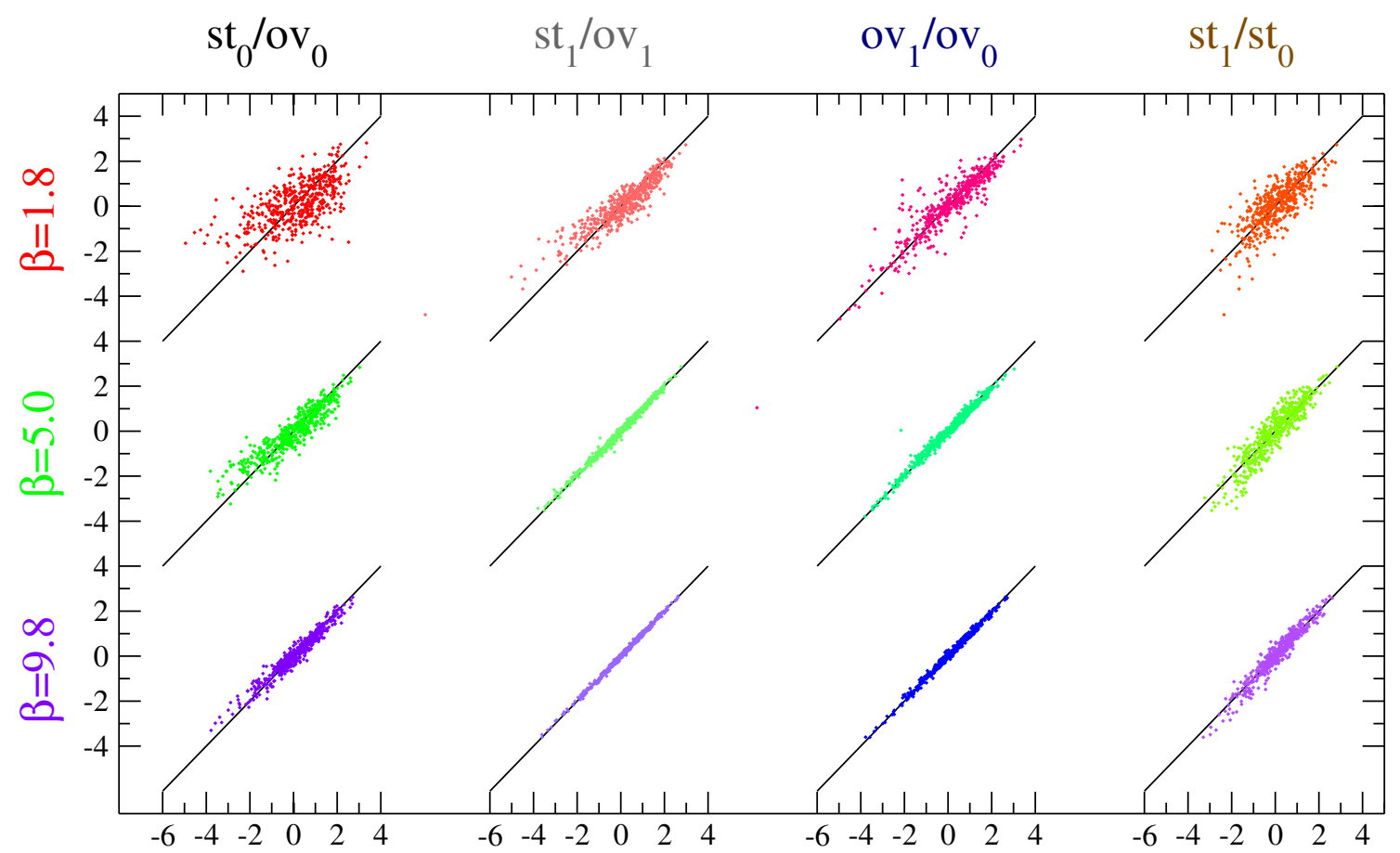

Figure 14: Scatter plot of $\frac{1}{2} \log \operatorname{det}\left(D_{\mathrm{st}, m}\right)$ versus $\log \operatorname{det}\left(D_{\mathrm{ov}, m}\right)$ in a box of fixed physical volume and with $m / g=0.1$ fixed. The first column is without filtering, the second with. For comparison the correlation of the overlap determinant with and without filtering is shown and ditto for the rooted staggered determinant, where the deviations are known to be $O\left(a^{2}\right)$ effects. The black lines represent the identity. Figure from [40].

determinant ratios (from a configuration $U$ to $U^{\prime}$ ) as seen by these formulations, i.e. whether

$$
\left.\left.\frac{\lambda_{1} \lambda_{2} \ldots}{\lambda_{1}^{\prime} \lambda_{2}^{\prime} \ldots}\right|_{\mathrm{ov}} \simeq \frac{\gamma_{1} \gamma_{2} \ldots}{\gamma_{1}^{\prime} \gamma_{2}^{\prime} \ldots}\right|_{\mathrm{st}}
$$

If such a relation can be shown, specifically if one can prove that the deviation from an exact identity is caused by $O\left(a^{2}\right)$ cut-off effects, one would know that the rooted staggered determinant creates the "same" ensemble as the overlap action.

In 2D it is rather easy to compute exact determinants, in particular if one has all eigenvalues on several ensembles in a matched physical volume on disk. This being the case with our Schwinger model studies, we included a correlation plot in [47, 40], see Fig. 14. The sign-flipped contribution to the effective action on typical quenched backgrounds, as seen by (rooted) staggered or (single) overlap fermions is plotted against each other. In either case the determinant has been normalized by an artificial reference configuration which realizes the ensemble mean in both directions, and the physical box volume and quark mass are the same in all graphs. On coarse lattices, there is a rather loose correlation, i.e. we see a "cigar" shaped cloud. The interesting observation is that the thickness of the "cigar" shrinks on finer lattices, i.e. the correlation improves towards the continuum. This is particularly obvious if the two operators are filtered (second column). The full line always represents the identity, not a fit. To interpret this plot it is useful to recall that a correlation between $S_{G}$ and $-\log \operatorname{det}\left(D_{\mathrm{ov}, m}\right)$ or $-\frac{1}{2} \log \operatorname{det}\left(D_{\mathrm{st}, m}\right)$ looks rather similar on coarse 
lattices, but here the correlation does not improve towards the continuum ${ }^{2}$. For comparison the third and fourth columns correlate different filtering levels for the overlap or staggered action, respectively. The point is that in these cases the difference is known to be an $O\left(a^{2}\right)$ effect. It is amusing to see that a filtered overlap determinant creates an ensemble that is closer to the one from a rooted staggered determinant at the same filtering level than to the one from an unfiltered overlap determinant. In summary, the similarity between the second and third column makes it plausible that $\operatorname{det}^{1 / N_{t}}\left(D_{\mathrm{st}, m}\right)$ and $\operatorname{det}\left(D_{\mathrm{ov}, m}\right)$ create ensembles that differ by cut-off effects only (a more quantitative analysis is included in [40]). To my knowledge Fig. 14 contains the strongest evidence available right now that the overlap operator $D_{\mathrm{ov}, m}$ might actually be (one version of) the desired "candidate" action $D_{\mathrm{ca}, m}$, i.e. that the conjectured identity

$$
\operatorname{det}\left(D_{\mathrm{ov}, m}\right)=\mathrm{const} \cdot \operatorname{det}^{1 / N_{t}}\left(D_{\mathrm{st}, m}\right)\left(1+O\left(a^{2}\right)\right)
$$

might hold true with $N_{t}=2^{d / 2}$ (it is understood that the two operators have the same renormalized mass). On the other hand, it could be that all this is just misleading. In that case the only lesson to be learned would be that numerical evidence - no matter how convincing it looks - is never good enough to decide on conceptual issues.

\section{Staggered Chiral Perturbation Theory}

The last piece of evidence in favor of the rooting procedure comes from fitting hadron data to functional forms specific to the the low-energy effective theory for staggered quarks. For a general review on effective theories at finite lattice spacing I refer to [48].

Let me first recall some of the foundations of standard Chiral Perturbation Theory (XPT) as formulated by Gasser and Leutwyler [49, 50]. The key observation is that in QCD at low energy (say below $1 \mathrm{GeV}$ ) chiral symmetry seriously constrains the interaction of "pions" $(\pi, K, \eta)$ with each other and with heavier degrees of freedom (e.g. nuclei, see the talk by U.-G. Meissner [51]). The way how this is implemented is through the Goldstone theorem [52, 53]. More specifically, they start from the fundamental assumption that in QCD with $N_{f}$ light quarks (the focus is on the meson sector with $N_{f}=2,3$ ) the pattern of spontaneous symmetry breaking (SSB) is

$$
S U\left(N_{f}\right)_{L} \times S U_{R}\left(N_{f}\right)_{R} \longrightarrow S U\left(N_{f}\right)_{V}
$$

and build the most general effective Lagrangian consistent with this (and a few more constraints like Lorentz covariance and parity conservation). In this framework an amplitude like, e.g. the $I=0$ $\pi \pi$ forward scattering amplitude $\left[s=0, v=(t-u) /\left(4 M_{\pi}\right)\right]$ has a low-momentum expansion

$$
F_{\pi \pi}(v)=T^{I=0}\left(0,2 M_{\pi}\left(M_{\pi}+v\right), 2 M_{\pi}\left(M_{\pi}-v\right)\right)=-\frac{M_{\pi}^{2}}{F_{\pi}^{2}}+O\left(p^{4}\right)
$$

in powers of $p^{2}$. As announced above, (8.2) tends to zero for $M_{\pi}^{2}, v \rightarrow 0$. The counting rule $p^{2} \sim m$ (where $m$ is identified with $M_{\pi}^{2}$ ) is dictated by the reaction to infinitesimally small breaking terms.

Given this background, it is clear that an extension of XPT to staggered quarks must build on an analysis how the broken taste symmetry at finite lattice spacing affects the pion mass. This was

\footnotetext{
${ }^{2}$ It simply means that there is a genuine unquenching effect that cannot be mimicked through a shift in $\beta$.
} 

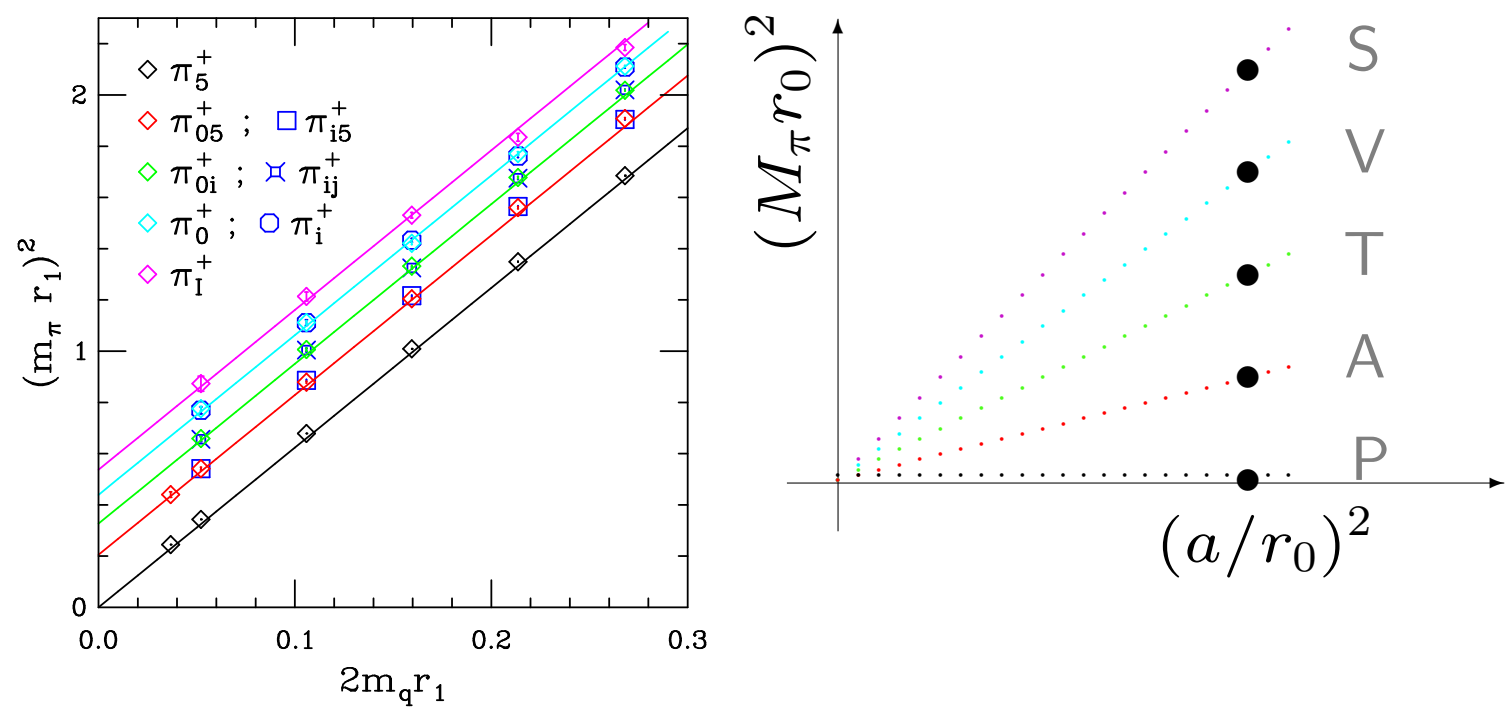

Figure 15: Left: $M_{\pi}^{2}$ versus $m$ for Goldstone pions (lowest line) and "non-Goldstone" pions (in parallel). Figure from [57]. Right: $M_{\pi}^{2}$ versus $a^{2}$ for Goldstone pions ("P") and "non-Goldstone" pions ("A,T,V,S").

done in a paper by Lee and Sharpe [54], and Fig. 15 illustrates their main findings. The generalized "pions" built, say, from an up-quark and a down-antiquark fall into different taste irreps $\bar{d}\left(\gamma_{5} \otimes \tau\right) u$. Only the taste pseudoscalar ( $\tau=\tau_{5}$, "P" in Fig. 15) is massless in the chiral limit at finite lattice spacing; this state is to be identified with the physical $\pi^{+}$. All taste non-P states ("A,T,V,S" in Fig. 15) stay massive in the chiral limit; the excess of their squared masses over the $\tau=\tau_{5}$ partner is nearly independent of the quark mass. Physical correlators project on taste pseudoscalars in the asymptotic states, but in intermediate loops all taste irreps do, of course, contribute. Even with intermediate flips into unphysical tastes being unavoidable, the continuum limit might be OK, if the contribution from physical intermediate states is far less than what continuum XPT would predict. The non-P mass excess diminishes like $a^{2}$ (see Fig. 15). Hence a scheme in which a cut in a 4-point function is built up from several contributions, each of which has a fraction of the continuum weight, would be OK if the fractions add up to 1 . In fact, such a desired behavior is built into the effective theory via an enlarged symmetry group [and a dedicated prescription]. The key assumption is as follows. With $N_{f}$ flavors of (4-taste) quark fields the pattern of SSB is

$$
S U\left(4 N_{f}\right)_{L} \times S U\left(4 N_{f}\right)_{R} \rightarrow S U\left(4 N_{f}\right)_{V}
$$

leading to $16 N_{f}^{2}-1$ pseudo-Goldstone bosons, collected in the $12 \times 12$ matrix (for $N_{f}=3$ )

$$
U=e^{\mathrm{i} \Phi / f} \quad \text { where } \quad \Phi=\left(\begin{array}{c}
\Phi_{u} \pi^{+} K^{+} \\
\pi^{-} \Phi_{d} K^{0} \\
K^{-} \bar{K}^{0} \Phi_{s}
\end{array}\right)=\sum_{a, b=1}^{9,16} \Phi^{a b} \frac{\lambda^{a}}{2} T^{b}
$$

that transforms as $U \rightarrow V_{L} U V_{R}^{\dagger}$ under chiral rotations with unitary $V_{L}, V_{R}$.

Staggered Chiral Perturbation Theory (SXPT) is the systematic extension of the Lee-Sharpe analysis to higher loop order, worked out in detail by Aubin and Bernard [55, 56]. The counting 
rule $p^{2} \sim m \sim a^{2}$ follows from the discussion above and the leading order Lagrangian reads

$$
L=\frac{f^{2}}{8} \operatorname{tr}\left(\partial_{\mu} U \partial_{\mu} U^{\dagger}\right)-\frac{\Sigma}{2} \operatorname{tr}\left(M U+M U^{\dagger}\right)+\frac{2 m_{0}^{2}}{3}\left(\Phi_{u, \mathrm{TS}}+\Phi_{d, \mathrm{TS}}+\Phi_{s, \mathrm{TS}}\right)+a^{2} V_{\mathrm{TB}}
$$

with $f \simeq 122 \mathrm{MeV}$ the pion decay constant in the chiral limit, $\Sigma \simeq(270 \mathrm{MeV})^{3}$ the chiral condensate [in ( $\overline{\mathrm{MS}}, 2 \mathrm{GeV})$ conventions] and $M=\operatorname{diag}\left(m_{u}, \ldots, m_{d}, \ldots, m_{s}, \ldots\right)$ the $12 \times 12$ quark mass matrix. From a look at $(8.4,8.5)$ it is clear that some details of the theory are rather different from ordinary XPT. The flavor sum in (8.4) extends up to 9 , not just 8 (here, $\lambda^{9}=\sqrt{2 / 3} I$ ). This is necessary, since (8.3) yields $12^{2}-1=143$ Goldstone and non-Goldstone bosons. With only $8 \cdot 16=128$ terms the dimensionality of the tangent space would be too small. On the other hand, with $9 \cdot 16=144$ terms there is space for one extra degree of freedom. This extra degree, the physical $\eta^{\prime}$, gets lifted through the piece with the $m_{0}^{2}$ prefactor in (8.5) which is designed to affect only the combined flavor and taste singlet state. That the $\eta^{\prime}$ is an explicit degree of freedom creates a similarity with quenched or partially quenched XPT, even for $m_{\mathrm{sea}}=m_{\mathrm{val}}$. The taste breaking potential $V_{\mathrm{TB}}$ in (8.5) implements the excess mass of the non-Goldstone pions that has been illustrated in Fig. 15. With this setup several important quantities have been calculated at 1-loop level, e.g. for $M_{\pi}, f_{\pi}, M_{K}, f_{K}$ it is known how they react to changes in $m$ and $a[55,56]$.

The SSB pattern (8.3) has been declared an assumption. Here, I would like to emphasize that the analogous pattern (8.1) remains an assumption in ordinary XPT, too. Of course, Gasser and Leutwyler mention that there is a wealth of evidence [from pions in the real world being so light, and even from the lattice] that the breaking (8.1) is actually realized in nature. The point is that their argument is evidence based, there is no proof that standard XPT is the correct low-energy theory of QCD. With SXPT the situation is rather analogous. One can compare the predictions to lattice data and see whether they fit. The MILC collaboration has done this in great detail, performing correlated fits in various circumstances (see e.g. [57]). Given the large number of free parameters, it is not so surprising that they get horrific covariance matrices, but in terms of the $\chi^{2} /$ d.o.f. obtained their fits are quite acceptable. Perhaps the most significant observation is that they cannot fit the taste pseudoscalar part of their data with continuum XPT, it is crucial to analyze their data in the full SXPT framework. Another point covered in [57] is that they generalize their formulae to allow for an arbitrary number of remaining tastes per flavor (not just 1). Fitting some of their lighter data with this extra parameter, they obtain 1.28(12) as the preferred number. More detailed tests have been proposed by Sharpe and van de Water [58], and experiences in more exotic sectors have been reported at this conference $[59,60]$. At this point there is a huge body of evidence that SXPT allows for an excellent description of a wealth of lattice data with the rooted staggered determinant. In principle there is, of course, the possibility that the "rooting trick" is incorrect and SXPT just a stunningly accurate model. Personally, I do not consider any accidental agreement of two flawed theories very likely, and this is why I rate the overall outcome of the MILC fits good evidence in favor of both the "rooting trick" and the SSB pattern (8.3). Still, the generic comment applies that even the best numerical evidence does not amount to a mathematical proof.

\section{Summary}

The question whether in QCD with $N_{f}=2+1$ light quarks the staggered action yields the right continuum limit remains unsolved. Instead of drawing any conclusion, let me just summarize: 
1. Full QCD with $N_{f}=2+1$ staggered fermions is controversial, since the Boltzmann weight $\operatorname{det}^{1 / 4}\left(D_{\text {st }}\right)$ assumes a taste symmetry which is only approximate.

2. Formally, the taste symmetry breaking is due to irrelevant interaction terms and should thus go away in the continuum limit.

3. Weak coupling, filtering, RG blocking reduce the taste splitting and give staggered quarks more appealing features, but there is no guarantee that no trace is left in the continuum.

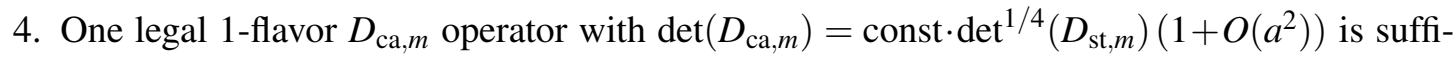
cient to re-interpret existing staggered ensembles as being generated with a local action.

5. Even if such a $D_{\mathrm{ca}, m}$ exists, the problem of (exact) unitarity in the fundamental theory remains when sticking to staggered spectroscopy. If the construction is exact, i.e. if there is no $O\left(a^{2}\right)$ term above, the problem is easily overcome by using this $D_{\text {ca }, m}$ in the valence sector.

Acknowledgments: I would like to thank Christian Hoelbling and Urs Wenger for a series of enjoyable collaborations and Christian for specific help in preparing the talk and for allowing me to show Fig. 12. In addition, I benefitted from discussions with G. Bali, C. Bernard, G. Colangelo, C. DeTar, K. Jansen, A. Kronfeld, F. Maresca, F. Niedermayer, Y. Shamir and S. Sharpe.

\section{References}

[1] J.B. Kogut and L. Susskind, Phys. Rev. D 11, 395 (1975).

[2] C.T.H. Davies et al. [HPQCD Collaboration], hep-lat/0304004.

[3] A. Patel and S.R. Sharpe, Nucl. Phys. B 395, 701 (1993) [hep-lat/9210039].

[4] S.R. Sharpe, hep-ph/9412243.

[5] H.S. Sharatchandra, H.J. Thun and P. Weisz, Nucl. Phys. B 192, 205 (1981).

[6] F. Gliozzi, Nucl. Phys. B 204, 419 (1982).

[7] A. Duncan, R. Roskies and H. Vaidya, Phys. Lett. B 114, 439 (1982).

[8] H. Kluberg-Stern, A. Morel, O. Napoly and B. Petersson, Nucl. Phys. B 220, 447 (1983).

[9] Y.B. Luo, Phys. Rev. D 55, 353 (1997) [hep-lat/9604025].

[10] T. DeGrand, Int. J. Mod. Phys. A 19, 1337 (2004) [hep-ph/0312241].

[11] E. Marinari, G. Parisi and C. Rebbi, Nucl. Phys. B 190, 734 (1981).

[12] See e.g. Sect. 1.6 in I. Montvay and G. Münster, "Quantum fields on a lattice", Cambridge (1994) and references cited therein, in particular $[1.49,1.50,1.51]$.

[13] K. Jansen, Nucl. Phys. Proc. Suppl. 129, 3 (2004) [hep-lat/0311039].

[14] P. Hernandez, K. Jansen and M. Lüscher, Nucl. Phys. B 552, 363 (1999) [hep-lat/9808010].

[15] B. Bunk, M. Della Morte, K. Jansen and F. Knechtli, Nucl. Phys. B 697, 343 (2004) [hep-lat/0403022].

[16] A. Hart and E. Muller, Phys. Rev. D 70, 057502 (2004) [hep-lat/0406030].

[17] D.H. Adams, hep-lat/0411030.

[18] F. Maresca and M. Peardon, hep-lat/0411029.

[19] P.H. Ginsparg and K.G. Wilson, Phys. Rev. D 25, 2649 (1982).

[20] Y. Shamir, Phys. Rev. D 71, 034509 (2005) [hep-lat/0412014]. 
[21] F. Maresca, these proceedings.

[22] J. Giedt, hep-lat/0507002.

[23] H. Neuberger, Phys. Rev. D 70, 097504 (2004) [hep-lat/0409144].

[24] T. DeGrand [MILC Collaboration], Phys. Rev. D 58, 094503 (1998) [hep-lat/9802012].

[25] T. Blum et al., Phys. Rev. D 55, 1133 (1997) [hep-lat/9609036].

[26] P. Lepage, Phys. Rev. D 59, 074502 (1999) [hep-lat/9809157].

[27] K. Orginos, D. Toussaint and R.L. Sugar [MILC Collaboration], Phys. Rev. D 60, 054503 (1999) [hep-lat/9903032].

[28] A. Hasenfratz and F. Knechtli, Phys. Rev. D 64, 034504 (2001) [hep-lat/0103029].

[29] T. DeGrand, A. Hasenfratz and T.G. Kovacs, Phys. Rev. D 67, 054501 (2003) [hep-lat/0211006].

[30] H. Neuberger, Phys. Lett. B 417, 141 (1998) [hep-lat/9707022].

[31] S. Dürr, C. Hoelbling and U. Wenger, Phys. Rev. D 70, 094502 (2004) [hep-lat/0406027].

[32] S. Dürr, C. Hoelbling and U. Wenger, hep-lat/0506027.

[33] A.Hasenfratz, http://www.rccp.tsukuba.ac.jp/lat03/Dat/OHP/a.hasenfratz.ps

[34] E. Follana, A. Hart and C.T.H. Davies [HPQCD Collaboration], Phys. Rev. Lett. 93, 241601 (2004) [hep-lat/0406010].

[35] E. Follana, Nucl. Phys. Proc. Suppl. 140, 141 (2005) [hep-lat/0409062].

[36] K.Y. Wong and R.M. Woloshyn, Phys. Rev. D 71, 094508 (2005) [hep-lat/0412001].

[37] E. Follana, A. Hart, C.T.H. Davies and Q. Mason [HPQCD Collaboration], hep-lat/0507011.

[38] J.S. Schwinger, Phys. Rev. 128, 2425 (1962).

[39] S. Dürr and C. Hoelbling, Phys. Rev. D 69, 034503 (2004) [hep-lat/0311002].

[40] S. Dürr and C. Hoelbling, Phys. Rev. D 71, 054501 (2005) [hep-lat/0411022].

[41] K.C. Bowler, B. Joo, R.D. Kenway, C.M. Maynard and R.J. Tweedie [UKQCD Collaboration], hep-lat/0411005.

[42] M. Golterman and Y. Shamir, Phys. Rev. D 71, 034502 (2005) [hep-lat/0411007].

[43] O. Bär, G. Rupak and N. Shoresh, Phys. Rev. D 67, 114505 (2003) [hep-lat/0210050].

[44] O. Bär, C. Bernard, G. Rupak and N. Shoresh, hep-lat/0503009.

[45] J. Smit and J.C. Vink, Nucl. Phys. B 286, 485 (1987).

[46] C. Bernard, Phys. Rev. D 71, 094020 (2005) [hep-lat/0412030].

[47] S. Dürr and C. Hoelbling, Nucl. Phys. Proc. Suppl. 140, 680 (2005) [hep-lat/0408039].

[48] O. Bär, Nucl. Phys. Proc. Suppl. 140, 106 (2005) [hep-lat/0409123].

[49] J. Gasser and H. Leutwyler, Annals Phys. 158, 142 (1984).

[50] J. Gasser and H. Leutwyler, Nucl. Phys. B 250, 465 (1985).

[51] U.-G. Meissner, these proceedings.

[52] J. Goldstone, Nuovo Cim. 19, 154 (1961).

[53] J. Goldstone, A. Salam and S. Weinberg, Phys. Rev. 127, 965 (1962).

[54] W.J. Lee and S.R. Sharpe, Phys. Rev. D 60, 114503 (1999) [hep-lat/9905023].

[55] C. Aubin and C. Bernard, Phys. Rev. D 68, 034014 (2003) [hep-lat/0304014].

[56] C. Aubin and C. Bernard, Phys. Rev. D 68, 074011 (2003) [hep-lat/0306026].

[57] C. Aubin et al. [MILC Collaboration], Phys. Rev. D 70, 114501 (2004) [hep-lat/0407028].

[58] S.R. Sharpe and R.S. Van de Water, Phys. Rev. D 71, 114505 (2005) [hep-lat/0409018].

[59] E.B. Gregory et al., these proceedings.

[60] S. Prelovsek, these proceedings. 